\title{
1 YTHDF1 drives intestinal immune response against bacterial infection
}

2 Xin Zong, ${ }^{1}$ Xiao Xiao, ${ }^{1}$ Bin Shen, ${ }^{3}$ Qin Jiang, ${ }^{1}$ Hong Wang, ${ }^{1}$ Zeqing Lu, ${ }^{1,2}$ Fengqin

3 Wang, ${ }^{1,2}$ Minliang Jing, ${ }^{1,2}$ Yizhen Wang, ${ }^{1,2, *}$

41 Key Laboratory of Molecular Animal Nutrition, Ministry of Education, College of

5 Animal Sciences, Zhejiang University, Hangzhou, China.

62 Key Laboratory of Animal Nutrition and Feed Science in Eastern China, Ministry of

7 Agriculture, College of Animal Sciences, Zhejiang University, Hangzhou, China.

83 State Key Laboratory of Reproductive Medicine, Department of Histology and

9 Embryology, Nanjing Medical University, Nanjing, China.

10 *Correspondence: 866 Yuhangtang Road, Hangzhou, Zhejiang Province 310058,

11 People's Republic of China. Tel: +86 571 88982815; Fax: +86 57188982650. E-mail

12 address: yzwang321@zju.edu.cn. 


\section{Abstract}

Invasion of pathogenic bacteria is a serious threat to intestinal health. Recent emerging evidence has demonstrated that N6-methyladenosine $\left(\mathrm{m}^{6} \mathrm{~A}\right)$ is closely associated with innate immunity; however, the underlying mechanism remains unclear. Herein, we aim to explore the function and mechanism of $\mathrm{m}^{6} \mathrm{~A}$ modification in the regulation of innate immune responses against bacterial pathogens in the intestine. Ribo-seq and $\mathrm{m}^{6} \mathrm{~A}$-seq data have demonstrated that YTHDF1, an $\mathrm{m}^{6} \mathrm{~A}$ reader, directs the translation of tumor necrosis factor receptor-associated factor 6 (TRAF6) mRNA to regulate immune responses via modulation of $\mathrm{m}^{6} \mathrm{~A}$ methylation near stop codon. Furthermore, we have identified a unique mechanism that the interaction between YTHDF1 and the host factor DDX60 are critical in regulating intestinal immune response against bacterial infection by recognizing TRAF6 target transcripts. Additionally, our results provide novel insights as to why YTHDF1 could recognize its unique targets using the same domain as other YTHDF proteins. This work identifies YTHDF1 as a key driver of intestinal immune responses and provides an avenue for development of novel strategies to modulate intestinal immune response against bacterial infection. 


\section{Introduction}

Enterotoxigenic Escherichia coli (ETEC) causes diarrhea by colonizing the small intestine, leading to substantial mortality and morbidity in children and animals (Qadri, Akhtar et al., 2019). Intestinal epithelial cells (IECs) constitute an integral component of a highly regulated communication network that senses microbial and environmental stimuli, as well as endogenous danger signals (Peterson \& Artis, 2014). Activation of an IEC-specific immune response induces the production of a myriad of cytokines, chemokines and acute phase proteins (Henderson, van Limbergen et al., 2011). It is well known that toll-like receptors (TLRs) and nuclear factor- $\kappa \mathrm{B}$ (NF- $\kappa \mathrm{B}$ ) signaling play important roles in cytokine and chemokine production to induce the epithelial immune defense, mobilize immune effector cells, and activate adaptive immunity (Price, Shamardani et al., 2018). Tumor necrosis factor receptor-associated factor 6 (TRAF6), one of the key regulators mediating TLR and subsequent NF- $\kappa B$ signaling, is triggered by complex stimuli, including bacterial infection (Walsh, Lee et al., 2015). However, the mechanisms that regulate immune responses in the intestine remain largely unexplored.

Recently, increasing evidence has indicated that RNA modifications provide a powerful means to dynamically regulate gene expression (Zhao, Roundtree et al., 2018). Methylation of adenosine at the N6 position $\left(m^{6} \mathrm{~A}\right)$ is the most prevalent known posttranscriptional modification in mammalian mRNA (Roundtree, Evans et al., 2017). Similar to DNA methylation, $m^{6} \mathrm{~A}$ modification is reversibly catalyzed by a different set of enzymes; the modification is generated by a stable core catalytic complex (METTL3/METTL14/WTAP) and can be removed by demethylase FTO or ALKBH5. This $\mathrm{m}^{6} \mathrm{~A}$ functions as a "reader" that directly binds to RNA in the cytoplasmic space (Zhang, Fu et al., 2019). To date, three major "reader" proteins, including those of the YTH domain family (YTHDF) 1, YTHDF2, and YTHDF3, have been demonstrated to recognize the $\mathrm{m}^{6} \mathrm{~A}$ site through their YTH domain (Shi, Wei et al., 2019). Among these, YTHDF1 is believed to interact with translation initiation factors during the translation of $\mathrm{m}^{6} \mathrm{~A}$-modified mRNA (Wang, Zhao et al., 2015), whereas YTHDF2 promotes the degradation of its target transcripts partially via recruitment of the CCR4-NOT deadenylase complex (Du, Zhao et al., 2016). YTHDF3 cooperates with both YTHDF1 and YTHDF2 to regulate the translation and decay of methylated mRNAs (Shi, Wang et al., 2017). However, the role(s) of YTHDF proteins in IEC-specific immune responses and the mechanism underlying the triggering of YTHDF protein by stimuli to promote translation or degradation remain unknown. Function of RNA $\mathrm{m}^{6} \mathrm{~A}$ modification as well as YTHDF proteins in intestinal immune responses is of interest; however, it has not been investigated in detail. 
DEAD-box (DDX) proteins, which contain a conserved Asp-Glu-Ala-Asp motif, are the largest family of RNA helicases (Banroques, Cordin et al., 2011). DDX proteins interact with both rRNAs and mRNAs to regulate multiple biological functions, such as translation initiation, mRNA synthesis, and RNA splicing (Fu, Wu et al., 2016). The DEAD-box helicase DDX60 was identified from virus-infected human dendritic cells (DCs) (Miyashita, Oshiumi et al., 2011); it is involved in a subset of innate immune pathways across various cell types (Goubau, van der Veen et al., 2015). In this study, we revealed that YTHDF1 is critical for intestinal immune responses against bacterial infection both in vitro and in vivo. Apparently, YTHDF1 ablation resulted in substantially reduced immune responses via downregulation of TRAF6 transcript translation. METTL3-mediated $\mathrm{m}^{6} \mathrm{~A}$ modifications, either within the 3'UTR region or the coding sequence (CDS), are involved in these events. We also showed that interaction with DDX60 is essential for YTHDF1 to recognize $\mathrm{m}^{6} \mathrm{~A}$ on TRAF6 transcripts and to direct its protein synthesis. Collectively, our findings highlighted an important role of YTHDF1 in protection against bacterial infection and maintenance of intestinal immune homeostasis. 


\section{Results}

\section{YTHDF1 is critical for IECs to defense against bacterial infection}

To evaluate the potential involvement of the YTHDF protein family in IECs upon bacterial infection, we first investigated cultured IPEC-J2 cells exposed to LPS and $E T E C$. We found that gene copy number of YTHDF1 was much higher than that of YTHDF2 or YTHDF3 after LPS stimulation or ETEC infection (Fig 1A), which indicated that YTHDF1 plays an important role in these events.

To determine the role of YTHDF1 in IECs' defense against bacterial infection, we silenced YTHDF1 expression in IPEC-J2 cells by lentiviral vector-delivered expression of short hairpin RNAs (Fig 1B and C). Remarkably, cytokine multiplex assays showed that LPS-induced cytokine production, including that of IL-1 $\beta$, IL-6, and TNF- $\alpha$, was substantially decreased under YTHDF1-deficient condition (Fig 1D and E). These results were verified by ELISA and qPCR (Fig 1F and G). Similarly, $E T E C$-induced expression of inflammatory cytokines, such as TNF- $\alpha$ and IL-6, were downregulated owing to YTHDF1 deficiency (Fig EV1A and B). This phenotype was also validated in the human Caco-2 cells (Fig EV1C). In addition, we conducted rescue experiments in YTHDF1-depleted IPEC-J2 cells to further confirm that the decreased cytokine signature was specific to YTHDF1 knockdown. As expected, we found that the reintroduction of YTHDF1, not the vector control, enhanced cytokine expression upon infection in YTHDF1-depleted cells (Fig 1H). Furthermore, we observed that depletion of YTHDF1 suppressed activation of the transcription factor $\mathrm{NF}-\kappa \mathrm{B}$ (p65) during LPS and ETEC stimulation, suggesting that YTHDF1 functions upstream of p65 (Fig EV2A and B). These data indicate that YTHDF1 is important for the immune response to combat infection in IECs, possibly via regulation of NF- $\mathrm{KB}$ signaling.

\section{YTHDF1 regulates IECs immune responses by directing translation of TRAF6}

YTHDF1 primarily functions in ribosomal loading of $\mathrm{m}^{6} \mathrm{~A}$-modified mRNAs to facilitate translation initiation (Wang et al., 2015). To explore the downstream targets of YTHDF1, we employed ribosome profiling to compare the translation efficiency profile in LPS-stimulated IPEC-J2 cells, either in the presence or absence of YTHDF1. As expected, compared with the scramble control cells, we found a notable decrease in translation efficiency, particularly for $\mathrm{m}^{6} \mathrm{~A}$-modified transcripts, in YTHDF1-knockdown IPEC-J2 cells (Fig 2A and B). A total of 2030 differentially translated mRNAs (947 upregulated and 1083 downregulated genes) were selected for gene KEGG pathway enrichment analysis (Fig 2C). We found that among the downregulated genes the top enriched pathways were involved in the following: "TNF signaling pathway," "Salmonella infection," and "NF- $\mathrm{kB}$ signaling pathway" 
(Fig EV3). Because previous results indicated that YTHDF1 might play a role in $\mathrm{NF}-\kappa \mathrm{B}$ signaling and is upstream of $\mathrm{p} 65$, we focused on the NF- $\kappa \mathrm{B}$ signaling pathway among the group of downregulated genes. We observed that the translation efficiency of TRAF6 mRNA was significantly decreased (Fig 2D), whereas that of I $\mathrm{KB} \alpha$ was merely affected (Fig EV4A). To directly demonstrate the subsequent translational outcomes of TRAF6, we performed a firefly luciferase (Fluc) reporter assay in IPEC-J2 cells in either the absence or presence of YTHDF1. Consistent with ribosome profiling, a significant decrease in TRAF6 translation was YTHDF1 is knockdown (Fig 2E). Furthermore, we analyzed the protein synthesis of TRAF6 with or without YTHDF1. Remarkably, TRAF6 synthesis was substantially reduced in the absence of YTHDF1 (Fig EV4B). Comparable levels of TRAF6 mRNA indicated that such reduced TRAF6 synthesis resulted from translational deficiency (Fig EV4B). Similar observations were also made following ETEC infection in human Caco-2 cells (Fig EV4C and D). Because TRAF6 is a key regulator in mediating TLR4 and subsequent signaling, we also analyzed the effect of YTHDF1 depletion on TLR4 signaling. Expectedly, downstream targets of TRAF6 such as IKK, JNK, and MAPK were suppressed, whereas those upstream of TRAF6 remained unchanged (Fig EV5). Cumulatively, we speculated that TRAF6 mRNA is one of the key targets of YTHDF1 in governing immune responses.

RNA binding activity is necessary for YTHDF1 to regulate translation. Thus, we performed the RNA immunoprecipitation (RIP)-qPCR to confirm the presence of this specific binding mechanism between YTHDF1 and TRAF6 mRNA. As expected, in contrast to the control immunoglobulin G (IgG), we detected abundant TRAF6 mRNA signal, but not for MyD88 and MAPK8, upon immunoprecipitation with YTHDF1 antibody (Fig 2F). These results indicated that the inactivation of MAPK signaling was due to suppressed TRAF6 expression, and was not a direct result of a lack of YTHDF1. Next, we constructed a YTHDF1-truncated plasmid and found that deletion of either the YTH domain $(\triangle \mathrm{YTH})$ or Pro/Gln/Asn-rich domain $(\Delta \mathrm{P} / \mathrm{Q} / \mathrm{N})$ of YTHDF1 abrogated its binding to TRAF6 mRNA (Fig 2G). Furthermore, all YTHDF1-truncated plasmid failed to rescue the decreased cytokine expression in YTHDF1-silenced IPEC-J2 cells (Fig EV6). These data suggest that YTHDF1 binds to TRAF6 mRNAs to initiate their translation and thereby regulate the expression of cytokines, and both the YTH and P/Q/N-rich domains of YTHDF1 are indispensable for effective YTHDF1-RNA interactions.

\section{YTHDF1-mediated translation of TRAF6 requires $\mathbf{m}^{6} \mathrm{~A}$ methylation}

As aforementioned, YTHDF1 is a "reader" of $\mathrm{m}^{6} \mathrm{~A}$. To test whether altered YTHDF1-sensitive gene expression is associated with $\mathrm{m}^{6} \mathrm{~A}$ modifications, we first compared $\mathrm{m}^{6} \mathrm{~A}$ abundance in scramble controls and YTHDF1-depleted IPEC-J2 cells 
with LPS stimulation. In contrast to the globally increased $\mathrm{m}^{6} \mathrm{~A}$ levels observed in the control cells upon LPS treatment, cells lacking YTHDF1 surprisingly showed no elevated levels of methylation (Fig EV7A), suggesting the involvement of mRNA methylation in YTHDF1-sensitive gene expression. Because YTHDF1 is not a methyltransferase, we determined the levels of methyltransferase and $\mathrm{m}^{6} \mathrm{~A}$ demethylase in cells exposed to LPS, which revealed that loss of YTHDF1 led to decreased levels of METTL3 but increased levels of FTO (Fig EV7B). Next, we mapped the $\mathrm{m}^{6} \mathrm{~A}$ methylomes in IPEC-J2 cells before and after infection using $\mathrm{m}^{6} \mathrm{~A}$ sequencing ( $\left.\mathrm{m}^{6} \mathrm{~A}-\mathrm{Seq}\right)$. We searched for consensus motifs and identified a GGACU sequence (Fig 3A), which was consistent with a previous report (Zhou, Wan et al., 2018). Among 240 substantially different methylated transcripts, 141 (including TRAF6) were upregulated, whereas the other 89 were downregulated (Fig 3B). In addition, similar pattern of $\mathrm{m}^{6} \mathrm{~A}$ distribution was observed in control and infected cells, regardless of whether the transcripts were upregulated or downregulated, with a majority of $\mathrm{m}^{6} \mathrm{~A}$ modifications being enriched near a stop codon and within the 3'UTR (Fig EV8A). Notably, we identified three statistically significant $\mathrm{m}^{6} \mathrm{~A}$ peaks in the TRAF6 transcripts, with two in the CDS vicinity of the stop codon and one representing a unique peak within the 3'UTR. All these peaks were substantially increased post-infection, suggesting functional relevance (Fig 3C). As expected, methylation levels of $\mathrm{m}^{6} \mathrm{~A}$, as indicated by MyD88 mRNA, showed no difference between control and infected cells (Fig EV8B). These observations suggest that $\mathrm{m}^{6} \mathrm{~A}$ modification on TRAF6 mRNAs is enhanced upon bacterial infection and is involved in its translation.

To further analyze the function of $\mathrm{m}^{6} \mathrm{~A}$ modification on TRAF6 mRNA, we created FLAG-tagged expression constructs containing the TRAF6 CDS with or without the 3'UTR (CDS-3'UTR) (Fig 3D). After transfection into TRAF6-silenced IPEC-J2 cells, we found that protein expression of both TRAF6 constructs was significantly increased in contrast to vector following ETEC infection (Fig 3D), suggesting that the 3'UTR or rather the $\mathrm{m}^{6} \mathrm{~A}$ within the 3'UTR was dispensable for YTHDF1-TRAF6 mRNA regulation. The enhanced expression of both TNF- $\alpha$ and IL- 6 induced by the forced expression of FLAG-tagged TRAF6 further confirmed this conclusion (Fig 3E). Together, these data indicate that TRAF6 protein synthesis depends on both YTHDF1 and $\mathrm{m}^{6} \mathrm{~A}$ modification in the CDS region of its mRNA.

Next, we introduced a mutation in the m6A sites (corresponding to the conserved m6A motif) on TRAF6 mRNA (Fig 3F) in the FLAG-TRAF6 plasmids. These constructs were then transfected into TRAF6-silenced IPEC-J2 cells. After ETEC infection, we found that the TRAF6 protein levels were remarkably increased in cells transfected with mut1-3, but not mut4 (all three m6A sites mutated) (Fig 3F). 
Consistently, in addition to the mut4 plasmid, the other three mutation plasmids had similar effects as wild-type TRAF6 plasmids upon inducing the expression of cytokines (Fig 3G). In short, in a bacterial immune response, $\mathrm{m}^{6} \mathrm{~A}$ near the stop codon, either within the CDS or 3'UTR, is capable of mediating the mRNA translation of TRAF6.

\section{DDX60 is a coregulator for YTHDF1 regulating TRAF6 synthesize}

To gain further insights into the mechanism of YTHDF1-TRAF6 mRNA action, we hypothesized that the presence of a coregulator is required for facilitating YTHDF1 to accurately recognize its targets and such an effector molecule is sensitive to the states of both YTHDF1 and $\mathrm{m}^{6} \mathrm{~A}$. As an alternative approach, we purified YTHDF1-interacted proteins using YTHDF1 antibody obtained from LPS-stimulated IPEC-J2 cells. We employed tandem mass spectrometry analysis (MS/MS) to identify and quantify the associated protein components. In total, 466 proteins were enriched by YTHDF1, with the top candidate being DDX60 (Fig 4A). Next, we directly confirmed the interaction between YTHDF1 and DDX60 by immunoprecipitation and identified an endogenous interaction between YTHDF1 and DDX60 in LPS-stimulated cells. Moreover, the interaction between YTHDF1 and DDX60 persisted even after RNA degradation by RNase (Fig 4B and C). This coregulator is sensitive to not only YTHDF1 but also $\mathrm{m}^{6} \mathrm{~A}$. To further confirm the possibility that DDX60 is the coregulator, the transcriptome was analyzed by mRNA sequencing (mRNA-seq) using RNA isolated from scramble control, YTHDF1-knockdown, and METTL3-knockdown IPEC-J2 cells. METTL3 is a core methyltransferase of $\mathrm{m}^{6} \mathrm{~A}$ and determines the global level of mRNA $\mathrm{m}^{6} \mathrm{~A}$ modification (Liu, Yue et al., 2014). DDX60 was analyzed in the DEGs sensitive to both YTHDF1 and METTL3, which revealed that DDX60 may act as a coregulator in YTHDF1- $\mathrm{m}^{6} \mathrm{~A}$ interaction(s) (Fig 4D). Furthermore, loss of YTHDF1 led to increased DDX60 protein level, which was consistent with the mRNA-seq data (Fig EV9A).

Based on the aforementioned findings, we speculated that DDX60 interacts with YTHDF1 and is involved in YTHDF1-TRAF6 regulation. To test this hypothesis, we created a DDX60-knockdown IPEC-J2 cell line (Fig 4E). Upon LPS stimulation, the DDX60 knockdown suppressed TRAF6 synthesis and blocked activation of the NF- $\kappa \mathrm{B}$ signaling pathway as compared with the scramble control (Fig 4F). We observed a similar phenotype in human Caco-2 cells and ETEC-infected IPEC-J2 cells (Fig EV9B and C). Moreover, decreased production and gene expression of TNF- $\alpha$ and IL-6 confirmed these observations (Appendix Fig EV9D-F). Surprisingly, in contrast to the YTHDF1 knockdown, the transcription of TRAF6 was also decreased in cells lacking DDX60 (Fig 4G). These observations indicate that DDX60 
may act as a coregulator via stabilization and transportation of TRAF6 mRNAs to ensure that YTHDF1 accurately recognizes TRAF6 mRNA.

\section{DDX60 facilitates of YTHDF1 to accurately recognize TRAF6 mRNA}

Based on the working model of DDX60, we further tested whether DDX60 interacts with TRAF6 transcripts. Similar to YTHDF1, the abundance of TRAF6 transcripts but not those of MyD88 and MAPK8 was enriched by DDX60 (Fig 4H). Next, we constructed additional plasmids expressing various truncated fragments of DDX60 (Fig 4I). RIP-qPCR revealed that DDX60 lacking the HELICc domain could no longer enrich TRAF6 transcripts (Fig 4I), indicating that this domain is involved in its RNA binding activity. Furthermore, neither the DEADc nor HELICc domains alone could rescue the suppressed expression of TRAF6 or the activation of p65 caused by deletion of DDX60 (Fig 4J). Further functional studies showed a noticeable increase in cytokine expression in DDX60-depleted cells that were transfected with truncated DDX60 plasmids containing DEADc and HELICc domains, but not in cells transfected with plasmids containing the DEADc or HELICc domains alone (Fig 4K). Overall, these results indicate that DDX60 binds to TRAF6 mRNA via its HELICc domain; however, both the DEADc and HELICc domains are required for effective DDX60-driven expression of TRAF6 proteins.

To investigate the role of DDX60 in terms of association of transcripts and YTHDF1, we first investigated the binding of YTHDF1 to TRAF6 mRNA in the absence or presence of DDX60. As shown in Fig 5A, DDX60 depletion abrogated the interaction between YTHDF1 and TRAF6 mRNA. Further, we transfected YTHDF1-containing plasmid into IPEC-J2 cells either depleted of YTHDF1 only or both YTHDF1 and DDX60. We found that YTHDF1 could not rescue TRAF6 expression and p65 activation in YTHDF1-depleted cells in the absence of DDX60 (Fig 5B). These results indicated that the function of YTHDF1 in TRAF6 synthesis depends on DDX60. In addition, similar results from the TRAF6 and NF- $\kappa$ B luciferase reporter assay confirmed this conclusion (Fig 5C). Mechanistically, we employed constructed plasmids expressing various truncated fragments of YTHDF1 and DDX60, which revealed that YTHDF1-DDX60 interaction required the P/N/Q rich domain of YTHDF1 and the DEAD domain of DDX60 (Fig 5D and E). Thus, we conclude that the $\mathrm{P} / \mathrm{Q} / \mathrm{N}$-rich domain of YTHDF1 and the DEAD domain of DDX60 are responsible for YTHDF1-DDX60 interaction and the function of YTHDF1 in TRAF6 synthesis.

\section{YTHDF1 deficiency impedes the intestinal immune response in vivo}

To further elucidate the function of YTHDF1 during the bacterial immune response, we created a targeted deletion of YTHDF1 in mice using the CRISPR/Cas9 system (Fig EV10A). Immunoblotting showed that the expression of YTHDF1 was abrogated 
277 in the jejunum of YTHDF1 ${ }^{-/-}$mice following infection (Fig EV10B). YTHDF1 ${ }^{-/}$and

$278 \mathrm{YTHDF}^{+/+}$(i.e., WT) mice with similar body weights were selected and challenged

279 with ETEC infection via oral gavage. Compared with $\mathrm{YTHDF}^{+/+}$mice, $\mathrm{YTHDF}^{-/-}$

280 mice exhibited considerably less weight loss (Fig 6A), which was also accompanied

281 by a higher survival rate (Fig 6B) and longer colon length (Fig 6C). We next

282 evaluated production of cytokines and found that ETEC infection induced increased

283 production of inflammatory cytokines in $\mathrm{YTHDF}^{+/+}$mice, which was largely

284 compromised in $\mathrm{YTHDF}^{-/-}$mice (Fig 6D). Histological analysis showed a

285 suppressed inflammatory response during ETEC infection accompanied by lower

286 clinical disease scores in $\mathrm{YTHDF}^{-/-}$mice as compared with $\mathrm{YTHDF}^{+/+}$mice (Fig

287 6E). Intestinal immune response is often accompanied by infiltration of inflammatory

288 cells. As shown in Fig 6F, compared with $\mathrm{YTHDF}^{+/+}$mice, YTHDF1 ${ }^{-/-}$mice

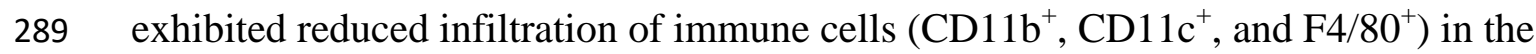

290 jejunum upon ETEC infection. This finding was further supported by

291 immunohistochemistry results in the colon (Fig EV10C). Finally, we evaluated

292 TRAF6 synthesis in vivo and found that the TRAF6 protein level in the jejunum and

293 colon from $\mathrm{YTHDF}^{-/-}$mice was significantly lower than the corresponding levels in

$294 \mathrm{YTHDF}^{+/+}$mice, albeit a similar level of TRAF6 mRNA was present (Fig 6G),

295 suggesting that YTHDF1 plays a significant role in TRAF6 synthesis in vivo. These

296 data convincingly indicate that YTHDF1 is vital to homeostatically sustain host

297 antibacterial immunity. 


\section{Discussion}

300 Epigenetic factors have provided new insight into the pathogenesis of immune

301 responses. Among many epigenetic modifications, RNA m ${ }^{6} \mathrm{~A}$ modification is one of the research focuses, which have shown to be related to innate immunity (Goubau et al., 2015, Hao, Hao et al., 2019, Li, Tong et al., 2017, Shi et al., 2017). However, the specific function of $\mathrm{m}^{6} \mathrm{~A}$ "readers" within the control of IEC immune responses and whether $\mathrm{m}^{6} \mathrm{~A}$ is involved in these events remain unclear. Bacteria activate the immune response of IECs by interacting with LPS, which is capable of passing through the intestinal barrier. In this study, we established models with LPS and ETEC mimicking the immune response in IECs following bacterial infection. We showed that YTHDF1 regulated IEC immune response by directing TRAF6 translation in an $\mathrm{m}^{6} \mathrm{~A}$ dependent manner. In addition, we identified DDX60 as a coregulator that facilitates YTHDF1 to recognize $m^{6} \mathrm{~A}$ on TRAF6 $\mathrm{mRNA}$.

The biological function of $\mathrm{m}^{6} \mathrm{~A}$ is mediated by its "readers". Previous study has shown that infection of human foreskin fibroblasts by cytomegalovirus (HCMV) upregulates the level of YTHDF2 (Winkler, Gillis et al., 2019). We have shown that YTHDF1 was much more sensitive to ETEC infection than YTHDF2 or YTHDF3.

\section{Our data showed that depletion of YTHDF1 led to a suppressed intestinal immune} response by blocking the NF- $\mathrm{\kappa B}$ signaling pathway both in vivo and in vitro. YTHDF1 was widely thought to enhance translation of its targets by interacting with initiation factors and facilitating ribosome loading (Wang et al., 2015). As revealed by Ribo-Seq data, the translation efficiency of $\mathrm{m}^{6} \mathrm{~A}$-modified transcripts was notably decreased in YTHDF1 knockdown IPEC-J2 cells in contrast to scramble control. We focused on the NF- $\mathrm{BB}$ signaling pathway, and observed that TRAF6 translation efficiency was significantly decreased, but not I $\mathrm{B} \alpha \mathrm{mRNA}$. Therefore, we speculated that TRAF6 mRNA was the target of YTHDF1 to govern the immune responses. However, a previous study showed that HCMV or dsDNA could trigger $\mathrm{m}^{6} \mathrm{~A}$-modified IFN $\beta$ production via the $\mathrm{m}^{6} \mathrm{~A}$ methyltransferase METTL3 and demethylase ALKBH5 (Rubio, Depledge et al., 2018). These results indicated that YTHDF1 could utilize two different mechanisms to regulate the IEC immune response. First, YTHDF1 regulated the translation of TRAF6 mRNA to control the activation of NF- $\kappa B$ signaling pathway. Second, YTHDF1 initiated the translation of $\mathrm{m}^{6} \mathrm{~A}$-modified effector molecule mRNAs, such as IFN $\beta$, to regulate their production directly.

\section{To test whether $\mathrm{m}^{6} \mathrm{~A}$ is involved in the IEC immune response, we measured} methylation levels in LPS-stimulated cells with or without YTHDF1. Interestingly, following YTHDF1 depletion no increase in methylation was observed, likely because YTHDF1 is an $\mathrm{m}^{6} \mathrm{~A}$ "reader" and not a methyltransferase. Decreased 
METTL3 and increased FTO levels in YTHDF1-knockdown cells suggested a feedback mechanism between YTHDF1 and $\mathrm{m}^{6} \mathrm{~A}$ levels, which also substantiated our hypothesis. We further confirmed and characterized the $\mathrm{m}^{6} \mathrm{~A}$ sites on TRAF6 mRNA by $\mathrm{m}^{6} \mathrm{~A}$-Seq. Consistent with the classical distribution of $\mathrm{m}^{6} \mathrm{~A}$ on the gene structure (Dominissini, Moshitch-Moshkovitz et al., 2012), methylation generating $\mathrm{m}^{6} \mathrm{~A}$ modifications on TRAF6 mRNA was concentrated in the vicinity of the stop codon at the conserved motif "RRACU". To clearly illustrate the mechanism by which YTHDF1- ${ }^{6}$ A participates in the IEC immune response, we constructed TRAF6 plasmids either with or without the 3'UTR region (designated CDS-3'UTR or CDS, respectively). To our surprise, both the TRAF6 CDS-3'UTR and CDS-only constructs successfully overexpressed TRAF6 protein. These results indicated that $\mathrm{m}^{6} \mathrm{~A}$ within the 3'UTR is not critically involved in YTHDF1-TRAF6 mRNA regulation. A similar finding was observed in TGF- $\beta$-induced SNAI1 expression, in which an $\mathrm{m}^{6} \mathrm{~A}$ in the 3'UTR had no effect on its protein synthesis (Lin, Chai et al., 2019). Mao et al. found that removing $\mathrm{CDS}^{6} \mathrm{~A}$ from transcripts resulted in a further decrease in translation (Mao, Dong et al., 2019). Therefore, these results improved the previous supposition that $\mathrm{m}^{6} \mathrm{~A}$ at the $3^{\prime} \mathrm{UTR}$ region mediated the regulatory role of YTHDF1 during translation (Wang et al., 2015, Zhang, Zhao et al., 2017). Taking advantage of variations of TRAF6 plasmids containing $\mathrm{m}^{6} \mathrm{~A}$ site mutations, we found that the $\mathrm{m}^{6} \mathrm{~A}$ modification was essential for TRAF6 protein synthesis but was not involved in its transcription. Considering the findings of the current study as well as the interaction between YTHDF1 and the EIF3 complex (Wang et al., 2015), we believe that a mediated loop structure formation is likely the key step for YTHDF1- ${ }^{6} \mathrm{~A}$ participation in translation, regardless of whether $\mathrm{m}^{6} \mathrm{~A}$ is within the $3^{\prime} \mathrm{UTR}$ or the CDS near the stop codon.

Structural and binding studies have suggested that the YTH domain is a specific $\mathrm{m}^{6} \mathrm{~A}$ "reader" (Xu, Wang et al., 2014, Zhu, Roundtree et al., 2014). We also found that YTHDF1 functioned by directly binding to TRAF6 transcripts at its YTH domain. However, our results suggest that loss of the $\mathrm{P} / \mathrm{Q} / \mathrm{N}$ rich domain failed to rescue YTHDF1 function in cytokine expression within YTHDF1-silenced IPEC-J2 cells, indicating that the intact YTHDF1 protein structure was required for TRAF6 protein synthesis. YTHDF family proteins share similar domain structures; however, in endometrial cancer cells YTHDF1 and YTHDF2 accurately target unique transcripts and regulate mRNA translation and decay, respectively (Shi et al., 2019). It is unknown as to how YTHDF1 or YTHDF2 recognize their specific $\mathrm{m}^{6} \mathrm{~A}$-marked targets accurately with the same domain. We hypothesized that a coregulator interacts with the P/Q/N-rich domain to ensure that each YTHDF1-TRAF6 transcript fulfills its specific role.

Based on the above hypothesis, we purified YTHDF1-interacted protein and 
376 identified the top candidates DDX60 as a coregulator in the YTHDF1-TRAF6

377 transcript interaction. Members of the DDX family of helicases broadly participate in

378 the innate immune response as coregulators. It has been shown that DDX46 proteins

379 bound to specific antiviral transcripts to negatively regulate the innate antiviral

380 response (Zheng, Hou et al., 2017). As expected, the relationship among YTHDF1,

381 DDX60, and TRAF6 was further confirmed through a YTHDF1 rescue assay in

382 DDX60 and YTHDF1 double knockdown IPEC-J2 cells. Mechanistically, our data

383 suggested that DDX60 binds to TRAF6 transcripts via its HELICc domain and

384 interacts with the P/Q/N-rich domain of YTHDF1 through its DEAD helicase domain,

385 which partially explains why YTHDF1 specifically recognizes its unique targets using

386 the same domain as other YTHDF proteins. However, consequently, decreased mRNA

387 level of TRAF6 was also observed in DDX60-depleted cells. We speculated that

388 DDX60 recognizes mRNA in the nucleus and functions as an RNA carrier, which

389 awaits further validation.

390 In summary, we identified a specific function and underlying mechanism for RNA

$391 \mathrm{~m}^{6} \mathrm{~A}$ modification in regulating the intestinal immune response via the complex of

392 YTHDF1, DDX60, and TRAF6 mRNA (Fig 7). This work not only provides novel

393 insights into the molecular mechanisms underlying IEC immune response but also

394 suggests novel strategies to cope with bacterial infections in the intestine. 


\section{Materials and Methods}

\section{Cell culture}

The porcine intestinal epithelial cell line IPEC-J2, human intestinal epithelial cell line Caco-2, and HEK 293T cells were purchased from the Cell Bank of the Chinese Academy of Sciences (Shanghai, China) and were cultured as described previously (Jiang, Sun et al., 2019, Zong, Zhao et al., 2019).

\section{Pathogens}

Escherichia coli strain O111:B4 LPS (Sigma-Aldrich) and enterotoxigenic Escherichia coli K88 (ETEC) were provided by Dr. W. Fang (Zhejiang University, China) and cultured in Luria-Bertani (LB) broth (Aoboxing, China) or on LB agar plates.

\section{Reagents}

Antibodies to the following were used in this study: anti-m ${ }^{6} \mathrm{~A}$ (Synaptic Systems, 202003), YTHDF1 (Proteintech, 66745-1-lg), TRAF6 (abcam, ab181622), $\beta$-actin (HuaAn, R1207-1), anti-Flag (Sigma-Aldrich, F3165), anti-Myc (abcam, ab32), DDX60 (abcam, ab139807), MyD88 (Proteintech, 23230-1-AP), phosphor-p65 (Cell Signaling Technology, 3033), phosphor-IкB $\alpha$ (Cell Signaling Technology, 2859), TRIF (Proteintech, 23288-1-AP), p65 (Proteintech, 10745-1-AP), IкB $\alpha$ (Proteintech, 10268-1-AP), TRAF3 (Proteintech, 18099-1-AP), phospho-IKK $\alpha / \beta$ (Cell Signaling Technology, 2078), IKK $\alpha$ (HuaAn, ER30911), IKK $\beta$ (HuaAn, ER1706-13), p38 (HuaAn, ET1602-26), phospho-p38 (Cell Signaling Technology, 9211), phospho-JNK (HuaAn, RT1488), JNK (HuaAn, RT1550), ERK1/2 (Cell Signaling Technology, 9102), phospho-ERK1/2 (HuaAn, ET1610-13), normal rabbit IgG (Cell Signaling Technology, 2729), and normal mouse IgG (Cell Signaling Technology, 5946).

\section{Mice and treatments}

All animal procedures were performed in accordance with the Guide for the Care and Use of Laboratory Animals in Zhejiang University. (Hangzhou, China). C57BL6/J mice (6-8 weeks old) were obtained from the Laboratory Animal Center of the Chinese Academy of Sciences (Shanghai, China). YTHDF1-deficient (YTHDF1 ${ }^{-/}$) mice on a C57BL/6J background were generated using CRISPR-Cas9 system, which were obtained from Bin Shen Laboratory in Nanjing Medical University (Nanjing, China). The oligonucleotides for sgRNAs were as follows: 1, TACCTGTCCAGTTACTATCC; 2, GGCACCATGGTCCACTGGAG. YTHDF1 ${ }^{+/+}$ and $\mathrm{YTHDF}^{-/-}$mice were infected with $100 \mu \mathrm{l}$ of the prepared bacterial suspension at a dose equivalent to $10^{9} \mathrm{CFU} /$ mouse by oral gavage. Total experimental period was 11 days. C57BL/6 mice were sacrificed at day 3 post-infection.

\section{Lentiviral short hairpin RNAs, siRNA and transfection}

YTHDF1- and DDX60-knockdown IPEC-J2 cells were generated by using lentiviral 
short hairpin (sh) RNAs (shRNAs); shRNA targeting sequences and siRNA are listed in Table EV1. Plasmids or siRNA were transfected into cells with Lipofectamine 2000 (Invitrogen) according to the manufacturer's instructions.

\section{Molecular cloning of related genes}

Related genomic cDNA were cloned from total RNAs extracted from IPEC-J2 cells by RT-PCR. FLAG-tagged YTHDF1 was cloned into pCMV-Tag 4 vector (Agilent, \#211174); Myc-tagged DDX60 was cloned into pRK-5 vector (BD PharMingen, 556104). We generated truncation plasmids based on full-length proteins. All other plasmids, including full-length, coding sequence, and mutation plasmids were generated by GenScript, which were then cloned into FLAG-pcDNA3.1 vector. All plasmids were confirmed by DNA sequencing. The corresponding primers used in this study are listed in Table EV1.

\section{RNA extraction, cDNA synthesis, and qPCR}

Total RNA was isolated using TRIzol reagent (Invitrogen), and poly(A) ${ }^{+}$mRNA was enriched from total RNA with GenElute mRNA Miniprep Kit (Sigma-Aldrich). The cDNA was reverse transcribed using M-MLV reverse transcriptase (Thermo Fisher Scientific) with $2 \mu \mathrm{g}$ of RNA from each sample. Next, quantitative PCR (qPCR) was performed as previously described (Zong et al., 2019). GAPDH or $\beta$-actin was used as endogenous control, and each reaction was run in triplicates. Gene copy numbers were then calculated using the following formula: number of copies $=($ DNA quantity $\left.\times 6.022 \times 10^{23}\right) /\left(\right.$ DNA length $\left.\times 1 \times 10^{9} \times 650\right)$. The primer sequences used for qPCR are listed in Supplementary Table EV1.

\section{RNA immunoprecipitation (RIP) assay}

RIP was performed according to the native RIP protocol as previously described (Gagliardi \& Matarazzo, 2016, Shen, Zhang et al., 2018). Briefly, cell lysates were prepared in polysome lysis buffer $(100 \mathrm{mM} \mathrm{KCl}, 5 \mathrm{mM} \mathrm{MgCl}, 10 \mathrm{mM}$ HEPES pH 7.0, and $0.5 \%$ NP40) supplemented with DTT, protease inhibitor cocktail (APExBIO), and RNase inhibitor (Promega) on ice. Cell lysates were incubated with antibody and protein A/G magnetic beads (Sigma) from $3 \mathrm{~h}$ to overnight at $4{ }^{\circ} \mathrm{C}$ under rotation conditions. Ten percent of the cell lysate supernatant was saved for input analysis. Beads were washed four times and the RNA released with proteinase $\mathrm{K}$ treatment for 30 min at $55^{\circ} \mathrm{C}$. Precipitated RNA was extracted and analyzed by qPCR. The primer sequences used are listed in Table EV1.

\section{$\mathrm{m}^{6} \mathrm{~A}$ dot blot}

The $\mathrm{m}^{6} \mathrm{~A}$ dot blots were conducted as previously described (Zhou, Wan et al., 2015, Zong et al., 2019). Equal amounts of poly(A) ${ }^{+}$mRNA was spotted on an Amersham Hybond-N+ membrane (GE Healthcare), followed by UV crosslinking. After blocking, the membrane was incubated with anti- $\mathrm{m}^{6} \mathrm{~A}$ antibody overnight at $4{ }^{\circ} \mathrm{C}$. The dots were visualized using a ChemiScope series 3400 Mini Imaging System. Methylene blue 
473 (Sigma-Aldrich) staining was used to show the amount of total RNA on the

474 membrane.

\section{Immunoblot}

476 Cells were lysed on ice in SDS-PAGE sample buffer [50 mM Tris (pH 6.8), $100 \mathrm{mM}$

477 DTT, 2\% SDS, $0.1 \%$ bromophenol blue, and 10\% glycerol]. Proteins were transferred 478 onto a polyvinylidene fluoride membrane (MilliporeSigma, Billerica, MA) and were 479 blotted and quantified as described previously (Zong et al., 2019).

\section{Luciferase reporter assays}

481 IPEC-J2 cells were plated in 24-well plates and then were transfected with pGL4.21

482 firefly luciferase reporter plasmid and Renilla luciferase plasmid, together with

483 various amounts of the appropriate control or protein-expressing plasmid(s). The

484 luciferase activity was measured using the Dual-Luciferase Reporter Assay System

485 (Promega). Reporter gene activity was determined by normalization of firefly

486 luciferase activity to Renilla luciferase activity.

487 Protein co-immunoprecipitation

488 The cell pellet was resuspended with 2 volumes of IP lysis buffer (Pierce)

489 supplemented with cocktail protease inhibitor (APExBIO), incubated on ice for

$49010 \mathrm{~min}$, and then incubated with primary antibody and Protein A/G magnetic beads at

$4914{ }^{\circ} \mathrm{C}$ overnight. The washed beads were resuspended in SDS sample buffer and

492 analyzed by immunoblotting or tandem mass spectrometry analysis.

\section{Tandem mass spectrometry analysis}

494 Peptide samples were analyzed using an Orbitrap Elite hybrid mass spectrometer

495 (Thermo Fisher) by Novogene (Beijing, China). Briefly, the purified protein samples

496 were separated by SDS-PAGE and visualized by silver staining. The proteins were

497 reduced, alkylated, and digested as previously described (Shevchenko, Tomas et al.,

498 2006). The extracted samples were dried, resuspended in $0.1 \%$ trifluoroacetic acid,

499 desalted with C18 ZipTips, dried again, and dissolved in $0.1 \%$ formic acid. Samples

500 were separated on a C18 analytical column using a two-solvent system.

\section{Cytokine multiplex assay}

502 Porcine cytokine antibody arrays (QAP-CYT-1) were obtained from and subsequently 503 conducted by RayBiotech (Guangzhou, China). Serum-free media from IPEC-J2

504 cultures or equivalent amounts of mouse intestinal tissue homogenate were used.

505 Intensity was normalized to internal positive controls for comparison.

\section{Cell treatments and ELISA}

507 The cells were cultured in six-well dishes until they reached approximately $80 \%$

508 confluence and then treated with LPS $(50 \mu \mathrm{g} / \mathrm{ml})$ or Escherichia coli K88 (MOI 10:1)

509 for varying durations. Untreated cells were used as controls. The concentration of 510 cytokines released into the supernatant or in intestines was measured via specific IL-6 or TNF- $\alpha$ ELISA (Quantikine ELISA Kit; Proteintech). 
512

513

514

515

516

517

518

519

520

521

522

523

524

525

526

527

528

529

530

531

532

533

534

535

536

537

538

539

540

541

542

543

544

545

546

547

548

549

550

\section{Histology and immunohistochemistry}

Excised intestine from euthanized mice was fixed in 4\% paraformaldehyde overnight at $4^{\circ} \mathrm{C}$. Samples were then sent to Servicebio (Wuhan, China) for histological and immunohistochemical analysis. Briefly, samples embedded in paraffin were deparaffinized and processed through a graded series of alcohol. Then, the sections were incubated with primary antibody followed by peroxidase-conjugated secondary antibody. Sections were scored by a blinded observer based on goblet cell depletion, leukocyte infiltration, and submucosal inflammation on a point scale of $0-3$, with 0 representing no pathology and 3 indicating the most severe pathology.

\section{Ribosome- protected fraction isolation, Ribo-Seq and analysis}

The ribosome-protected fraction isolation, library construction, high-throughput sequencing and analysis were performed by Novogene (Beijing, China). In brief, ribosome-protected mRNA was obtained by MNase digestion and RNA purification (Ingolia, Brar et al., 2012, Reid, Shenolikar et al., 2015). In total, $3 \mu \mathrm{g}$ of ribosome-protected mRNA was used to prepare a library with the NEBNext Small RNA Library Prep Set (New England Biolabs) according to the manufacturer's instructions. After PCR amplification, the samples were used for quality control and deep sequencing with an Illumina HiSeq 2000.

\section{$m^{6} A$ sequencing and analysis}

Library preparation of $\mathrm{m}^{6} \mathrm{~A}$ Sequencing and high-throughput sequencing were performed by Novogene (Beijing, China). The $\mathrm{m}^{6} \mathrm{~A}$ immunoprecipitation and preparation of libraries were performed as previously described (Dominissini et al., 2012). Purified RNA fragments were used for library construction with the NEBNext Ultra RNA Library Prep Kit and were sequenced with Illumina HiSeq 2000.

Sequencing reads were aligned to the Sscrofa11.1 by BWA, and the $\mathrm{m}^{6} \mathrm{~A}$ peak calling was performed by MACS2.

\section{RNA sequencing and analysis}

Library preparation of RNA sequencing and high-throughput sequencing were performed by Novogene (Beijing, China). Raw data were filtered, corrected, and mapped to locust genome sequences using the HISAT software. Gene expression levels were measured using the criteria of reads per $\mathrm{kb}$ per million mapped reads. DEGs were detected using the DESeq software (Trapnell, Williams et al., 2010). Genes with an adjusted $P$-value of $<0.05$ were described as being differentially expressed. Kyoto Encyclopedia of Genes and Genomes (KEGG) enrichment was performed by the Clusterfile software. Significance analysis was performed by Fisher's Exact Test.

\section{Statistical analysis}

Results were expressed as means \pm s.e.m. For multiple groups, one-way ANOVA was used to determine statistical differences, and t-tests were employed between two 
551 groups; ${ }^{*} P<0.05,{ }^{* *} P<0.01$. Data were analyzed with GraphPad Prism Software.

552 Each experiment was performed at least in triplicates unless otherwise stated.

\section{Acknowledgements}

554 The authors thank the Electronic Microscopy Center and the Agricultural, Biological,

555 and Environmental Test Center of Zhejiang University for assistance with the

556 confocal microscopy work. The authors thank Shu Zhu's lab in Zhejiang University

557 for assistance with the ETEC infection. This work was supported by the National

558 Natural Science Foundation of China (Grants 31630075).

559 Author contributions

560 X.Z and Y.Z.W designed the research; X.X, Q.J, H.W performed experiments and

561 collected data; X.Z wrote the manuscript; B.S and F.Q.W supported technical; Z.Q.L

562 and M.L.J reviewed the manuscript.

\section{Conflict of interest}

564 The authors declare that they have no competing interests.

565

566 


\section{References}

Banroques J, Cordin O, Doere M, Linder P, Tanner NK (2011) Analyses of the Functional Regions of DEAD-Box RNA "Helicases" with Deletion and Chimera Constructs Tested In Vivo and In Vitro. $J$ Mol Biol 413: 451-472

Dominissini D, Moshitch-Moshkovitz S, Schwartz S, Salmon-Divon M, Ungar L, Osenberg S, Cesarkas K, Jacob-Hirsch J, Amariglio N, Kupiec M, Sorek R, Rechavi G (2012) Topology of the human and mouse m(6)A RNA methylomes revealed by m(6)A-seq. Nature 485: 201-U84

Du H, Zhao Y, He JQ, Zhang Y, Xi HR, Liu MF, Ma JB, Wu LG (2016) YTHDF2 destabilizes m(6)A-containing RNA through direct recruitment of the CCR4-NOT deadenylase complex. Nat Commun 7

Fu TY, Wu CN, Sie HC, Cheng JT, Lin YS, Liou HH, Tseng YK, Shu CW, Tsai KW, Yen LM, Tseng HW, Tseng CJ, Ger LP, Liu PF (2016) Subsite-specific association of DEAD box RNA helicase DDX60 with the development and prognosis of oral squamous cell carcinoma. Oncotarget 7: 85097-85108

\section{Gagliardi M, Matarazzo MR (2016) RIP: RNA Immunoprecipitation. Methods Mol Biol 1480: 73-86}

Goubau D, van der Veen AG, Chakravarty P, Lin RT, Rogers N, Rehwinkel J, Deddouche S, Rosewell I, Hiscott J, Sousa CRE (2015) Mouse superkiller-2-like helicase DDX60 is dispensable for type I IFN induction and immunity to multiple viruses. Eur J Immunol 45: 3386-3403

Hao HJ, Hao SJ, Chen HH, Chen Z, Zhang YF, Wang J, Wang HZ, Zhang B, Qiu JM, Deng F, Guan WX (2019) N-6-methyladenosine modification and METTL3 modulate enterovirus 71 replication. Nucleic Acids Res 47: 362-374

Henderson P, van Limbergen JE, Schwarze J, Wilson DC (2011) Function of the Intestinal Epithelium and Its Dysregulation in Inflammatory Bowel Disease. Inflamm Bowel Dis 17: 382-395 Ingolia NT, Brar GA, Rouskin S, McGeachy AM, Weissman JS (2012) The ribosome profiling strategy for monitoring translation in vivo by deep sequencing of ribosome-protected mRNA fragments. Nat Protoc 7: 1534-50

Jiang Q, Sun BF, Liu Q, Cai M, Wu RF, Wang FQ, Yao YX, Wang YZ, Wang XX (2019) MTCH2 promotes adipogenesis in intramuscular preadipocytes via an m(6)A-YTHDF1-dependent mechanism. Faseb J 33: 2971-2981

Li HB, Tong JY, Zhu S, Batista PJ, Duffy EE, Zhao J, Bailis W, Cao GC, Kroehling L, Chen YY, Wang G, Broughton JP, Chen YG, Kluger Y, Simon MD, Chang HY, Yin ZN, Flavell RA (2017) m(6)A mRNA methylation controls T cell homeostasis by targeting the IL-7/STAT5/SOCS pathways. Nature 548: 338-+

Lin XY, Chai GS, Wu YM, Li JX, Chen F, Liu JZ, Luo GZ, Tauler J, Du J, Lin SB, He C, Wang HS (2019) RNA m(6)A methylation regulates the epithelial mesenchymal transition of cancer cells and translation of Snail. Nat Commun 10

Liu JZ, Yue YN, Han DL, Wang X, Fu Y, Zhang L, Jia GF, Yu M, Lu ZK, Deng X, Dai Q, Chen WZ, He C (2014) A METTL3-METTL14 complex mediates mammalian nuclear RNA N-6-adenosine methylation. Nat Chem Biol 10: 93-95

Mao YH, Dong LM, Liu XM, Guo JY, Ma HH, Shen B, Qian SB (2019) m(6)A in mRNA coding regions promotes translation via the RNA helicase-containing YTHDC2. Nat Commun 10

Miyashita M, Oshiumi H, Matsumoto M, Seya T (2011) DDX60, a DEXD/H Box Helicase, Is a Novel Antiviral Factor Promoting RIG-I-Like Receptor-Mediated Signaling. Molecular and Cellular Biology 


\section{1: 3802-3819}

Peterson LW, Artis D (2014) Intestinal epithelial cells: regulators of barrier function and immune homeostasis. Nature Reviews Immunology 14: 141-153

Price AE, Shamardani K, Lugo KA, Deguine J, Roberts AW, Lee BL, Barton GM (2018) A Map of Toll-like Receptor Expression in the Intestinal Epithelium Reveals Distinct Spatial, Cell Type-Specific, and Temporal Patterns. Immunity 49: 560-+

Qadri F, Akhtar M, Bhuiyan TR, Chowdhury MI, Ahmed T, Rafique TA, Khan A, Rahman SIA, Khanam F, Lundgren A, Wiklund G, Kaim J, Lofstrand M, Carlin N, Bourgeois AL, Maier N, Fix A, Wierzba T, Walker RI, Svennerholm AM (2019) Safety and immunogenicity of the oral, inactivated, enterotoxigenic Escherichia coli vaccine ETVAX in Bangladeshi children and infants: a double-blind, randomised, placebo-controlled phase 1/2 trial. Lancet Infect Dis

Reid DW, Shenolikar S, Nicchitta CV (2015) Simple and inexpensive ribosome profiling analysis of mRNA translation. Methods 91: 69-74

Roundtree IA, Evans ME, Pan T, He C (2017) Dynamic RNA Modifications in Gene Expression Regulation. Cell 169: 1187-1200

Rubio RM, Depledge DP, Bianco C, Thompson L, Mohr I (2018) RNA m(6)A modification enzymes shape innate responses to DNA by regulating interferon beta. Gene Dev 32: 1472-1484

Shen QC, Zhang Q, Shi Y, Shi QZ, Jiang YY, Gu Y, Li ZQ, Li X, Zhao K, Wang CM, Li N, Cao XT (2018) Tet2 promotes pathogen infection-induced myelopoiesis through mRNA oxidation. Nature 554: $123-+$

Shevchenko A, Tomas H, Havlis J, Olsen JV, Mann M (2006) In-gel digestion for mass spectrometric characterization of proteins and proteomes. Nat Protoc 1: 2856-2860

Shi H, Wei J, He C (2019) Where, When, and How: Context-Dependent Functions of RNA Methylation Writers, Readers, and Erasers. Mol Cell 74: 640-650

Shi HL, Wang X, Lu ZK, Zhao BXS, Ma HH, Hsu PJ, Liu C, He C (2017) YTHDF3 facilitates translation and decay of N-6-methyladenosine-modified RNA. Cell Research 27: 315-328

Trapnell C, Williams BA, Pertea G, Mortazavi A, Kwan G, van Baren MJ, Salzberg SL, Wold BJ, Pachter L (2010) Transcript assembly and quantification by RNA-Seq reveals unannotated transcripts and isoform switching during cell differentiation. Nat Biotechnol 28: 511-U174

Walsh MC, Lee J, Choi Y (2015) Tumor necrosis factor receptor- associated factor 6 (TRAF6) regulation of development, function, and homeostasis of the immune system. Immunol Rev 266: 72-92

Wang X, Zhao BS, Roundtree IA, Lu ZK, Han DL, Ma HH, Weng XC, Chen K, Shi HL, He C (2015) N-6-methyladenosine Modulates Messenger RNA Translation Efficiency. Cell 161: 1388-1399

Winkler R, Gillis E, Lasman L, Safra M, Geula S, Soyris C, Nachshon A, Tai-Schmiedel J, Friedman N, Le-Trilling VTK, Trilling M, Mandelboim M, Hanna JH, Schwartz S, Stern-Ginossar N (2019) m(6)A modification controls the innate immune response to infection by targeting type I interferons. Nature Immunology 20: 173-+

Xu C, Wang X, Liu K, Roundtree IA, Tempel W, Li YJ, Lu ZK, He C, Min JR (2014) Structural basis for selective binding of m(6)A RNA by the YTHDC1 YTH domain. Nat Chem Biol 10: 927-929

Zhang CY, Fu JR, Zhou YF (2019) A Review in Research Progress Concerning m6A Methylation and Immunoregulation. Front Immunol 10

Zhang SC, Zhao BS, Zhou AD, Lin KY, Zheng SP, Lu ZK, Chen YH, Sulman EP, Xie KP, Bogler O, Majumder S, He C, Huang SY (2017) m(6)A Demethylase ALKBH5 Maintains Tumorigenicity of Glioblastoma Stem-like Cells by Sustaining FOXM1 Expression and Cell Proliferation Program. 
Cancer Cell 31: 591-+

Zhao BS, Roundtree IA, He C (2018) Post-transcriptional gene regulation by mRNA modifications (vol 18,pg 31, 2017). Nat Rev Mol Cell Bio 19: 808-808

Zheng QL, Hou J, Zhou Y, Li ZY, Cao XT (2017) The RNA helicase DDX46 inhibits innate immunity by entrapping m(6)A-demethylated antiviral transcripts in the nucleus. Nature Immunology 18: 1094-+ Zhou J, Wan J, Gao XW, Zhang XQ, Jaffrey SR, Qian SB (2015) Dynamic m(6)A mRNA methylation directs translational control of heat shock response. Nature 526: 591-U332

Zhou J, Wan J, Shu XE, Mao YH, Liu XM, Yuan X, Zhang XQ, Hess ME, Bruning JC, Qian SB (2018) N-6-Methyladenosine Guides mRNA Alternative Translation during Integrated Stress Response. Mol Cell 69: 636-+

Zhu TT, Roundtree IA, Wang P, Wang X, Wang L, Sun C, Tian Y, Li J, He C, Xu YH (2014) Crystal structure of the YTH domain of YTHDF2 reveals mechanism for recognition of N6-methyladenosine. Cell Research 24: 1493-1496

Zong X, Zhao J, Wang H, Lu ZQ, Wang FQ, Du HH, Wang YZ (2019) Mettl3 Deficiency Sustains Long-Chain Fatty Acid Absorption through Suppressing Traf6-Dependent Inflammation Response. $J$ Immunol 202: 567-578 
Fig 1. YTHDF1 mediated the bacterial immune response in IECs.

674

(A) Gene copy numbers of YTHDF1, YTHDF2, and YTHDF3 after LPS stimulation or ETEC infection.

(B-C) The diminished effects of an established YTHDF1 depletion in IPEC-J2 cells; qPCR analysis of mRNA level (B); immunoblot analysis of protein level (C). NC, cells transfected with negative control scramble shRNA; shYthdf1, cells transfected with YTHDF1 shRNA [one of two shRNA constructs $(1,2)$ ].

(D) Laser scanning map of the cytokine microarray for LPS-treated indicated cells. Each cytokine was arrayed in quadruplicates (left table indicates cytokine map). Dots on the top represent positive controls.

(E) The relative fold change by signal intensity from (D). The results are relative to those of sh-Scramble group.

(F-G) ETEC-infected IPEC-J2 cells with $3 \mathrm{~h}$ treatment, cytokines in supernatants (F), and mRNA levels $(\mathrm{G})$ were determined.

(H) TNF- $\alpha$ and IL-6 mRNA levels in YTHDF1-depleted IPEC-J2 cells added back with the YTHDF1 plasmids. The qPCR results are presented relative to those of GAPDH. The data are expressed as the mean \pm s.e.m.; ${ }^{*} P<0.05,{ }^{* *} P<0.01, \mathrm{n}=3$ biological replicates.

\section{Fig 2. YTHDF1 maintained TRAF6 synthesis by directing its translation initiation.}

(A) The cumulative distribution of the fold change showing the translation efficiency between LPS-treated sh-Scramble (sh-Scram ${ }_{\mathrm{TE}}$ ) and shDF1 (sh-DF1 $1_{\mathrm{TE}}$ ) IPEC-J2 cells for $\mathrm{m}^{6} \mathrm{~A}$ targets (red), non- $\mathrm{m}^{6} \mathrm{~A}$ targets (green), and total (blue) mRNA. $P$-values were calculated using a two-sided Kolmogorov-Smirnov test; $\mathrm{n}=2$ independent biological replicates. (B) Box plot depicts the cumulative distribution of (A). Boxplot elements: center line, median; box limits, upper and lower quartiles; whiskers, 1\%-99\%. (C) Volcano plots of genes with differential translation efficiency from the same samples as in (A). The $P$-values were calculated with a two-sided likelihood ratio test and adjusted by the Benjamini-Hochberg method. (D) Genome-wide ribosome profiling of ribosome-protected mRNA fragments for TRAF6 in LPS-treated IPEC-J2 cells with or without YTHDF1 (sh-Scram or sh-DF1, respectively). Scales represent genomic RNA size and structure. (E) Luciferase activity of TRAF6 in IPEC-J2 cells after transfection with the indicated plasmids. Luciferase activity is presented relative to Renilla luciferase activity. (F) The interaction between YTHDF1 and TRAF6 
transcripts was measured by RNA immunoprecipitation (RIP)-qPCR and normalized to the input levels. Immunoblot of YTHDF1 (top right) showed that YTHDF1 antibody was immunoprecipitated successfully. (G) RIP-qPCR analyzed TRAF6 and GAPDH abundance immunoprecipitated by Flag antibody from the IPEC-J2 cells overexpressing YTHDF1, $\triangle \mathrm{YTH}$, and $\Delta \mathrm{P} / \mathrm{Q} / \mathrm{N}$ plasmid. The data are expressed as the mean \pm s.e.m.; ${ }^{* *} P<0.01,{ }^{* * *} P<0.001, \mathrm{n}=3$ biological replicates.

\section{Fig 3. Translation of TRAF6 was dependent on $\mathbf{m}^{6} \mathrm{~A}$ methylation.}

(A) Sequence motifs within $\mathrm{m}^{6} \mathrm{~A}$ peaks identified by using Homer software. (B) Analysis of differential $\mathrm{m}^{6} \mathrm{~A}$-modified gene in either uninfected IPEC-J2 cells or those infected with ETEC. (C) RNA-Seq of input RNA and $m^{6} \mathrm{~A}$ immunoprecipitated (IP) RNA in replicates for the TRAF6 transcript. The $m^{6} \mathrm{~A}$ motif sequences that correspond to an immunoprecipitate-enriched region are marked in green. (D) Rescue assay of specific TRAF6 plasmid in LPS-treated TRAF6-silenced IPEC-J2 cells. The left panel shows strategies of various TRAF6 plasmid construction. All plasmids were cloned into the FLAG-pcDNA3.1 vector. The right panel shows relative protein levels quantified by densitometry and normalized to the level of GFP. (E) TNF- $\alpha$ and IL-6 mRNA levels in TRAF6-depleted IPEC-J2 cells added back to the specific TRAF6 plasmid from (D). (F) Immunoblot analysis of FLAG-TRAF6, p65, and p-p65 in TRAF6-depleted IPEC-J2 cells transfected with specific TRAF6 plasmids and treated with $50 \mu \mathrm{g} / \mathrm{ml}$ LPS for $6 \mathrm{~h}$. The left panel shows the mutation strategy of the $\mathrm{m}^{6} \mathrm{~A}$ sites in TRAF6 mRNA. All plasmids were cloned into the FLAG-pcDNA3.1 vector. (G) TNF- $\alpha$ and IL-6 mRNA level in TRAF6-depleted IPEC-J2 cells were added back to the various TRAF6 plasmid from $(\mathrm{F})$. The qPCR results are presented relative to those of GAPDH. The data are expressed as the mean \pm s.e.m.; ${ }^{* *} P<0.01$, n.s., not significant, $\mathrm{n}=3$ biological replicates.

\section{Fig 4. DDX60 involved in TRAF6 protein synthesis.}

(A) Volcano plot of the $\log _{10}$ (P-value) versus $\log _{2}$ fold change (YTHDF1-IP/ IgG-IP) of MS-identified proteins from two biological replicates. Intensities were obtained from a MaxQuant database search. (B-C) Immunoprecipitation was performed with antibody to YTHDF1 (B) or DDX60 (B) and control IgG. After immunoprecipitation, samples were washed and incubated with RNase as indicated. The samples were analyzed by immunoblot assay using anti-YTHDF1 and anti-DDX60 antibodies. (D) Significantly regulated genes $(P<0.05)$ from overlapped YTHDF1 knockdown and METTL3 knockdown IPEC-J2 cells are presented in a 
scatter plot showing the genes that were commonly upregulated or downregulated following either YTHDF1 or METTL3 silencing (highlighted in green and blue, respectively). (E) Immunoblot analysis of DDX60 in IPEC-J2 cells transfected with negative control shRNA (NC) or DDX60-specific shRNA (one of three shRNA constructs: 1, 2, or 3). (F) Immunoblot analysis of TRAF6 and its downstream targets in indicated cells after LPS stimulation. The right panel shows the relative protein levels of TRAF6 quantified by densitometry and normalized to the level of $\beta$-actin. (E) Interaction between DDX60 and TRAF6 transcripts was measured by RIP-qPCR and normalized to the input levels. Immunoblot of DDX60 (top right) showed that DDX60 antibody was immunoprecipitated successfully. (F) The fold enrichment of TRAF6 and GAPDH mRNA as determined by RIP-qPCR in IPEC-J2 cells overexpressed the indicated plasmids. Schematic structure of Myc-DDX60 and the truncation strategy is shown above. $(\mathrm{G})$ The shDDX60 cells added back to the indicated plasmids upon LPS stimulation. The protein level of TRAF6, p65, and p-p65 were determined by immunoblot. The right panel shows the relative protein levels quantified by densitometry and normalized to the quantity of $\beta$-actin. The qPCR results are presented in relation to those of GAPDH. The data are expressed as the mean \pm s.e.m.; ${ }^{* *} P<0.01,{ }^{* * *} P<0.001$, n.s., not significant, $\mathrm{n}=3$ biological replicates.

\section{Fig 5. Interaction with DDX60 was critical for the role of YTHDF1 in TRAF6 protein synthesis.}

(A) RIP-qPCR analysis of the interaction between YTHDF1 and TRAF6 transcripts in IPEC-J2 cells with or without DDX60. (B) Rescue assay of YTHDF1 plasmid in LPS-treated YTHDF1 knockdown IPEC-J2 cells in the presence or absence of DDX60. (C) Luciferase activity of TRAF6 and NF- $\kappa$ B after co-transfection with the indicated plasmids in YTHDF1 knockdown IPEC-J2 cells subjected to DDX60 siRNA treatment. Luciferase activity is presented relative to Renilla luciferase activity. The data are expressed as the mean \pm s.e.m.; ${ }^{* *} P<0.01, \mathrm{n}=3$ biological replicates. (D-E) FLAG-tagged YTHDF1 partial fragments and/or Myc-tagged DDX60 expression vectors (D) or Myc-tagged DDX60 partial fragments and/or FLAG-tagged YTHDF1 expression vectors (E) were transfected into IPEC-J2 cells. After LPS stimulation for $6 \mathrm{~h}$, immunoprecipitation was performed with Flag or Myc antibodies.

\section{Fig 6. YTHDF1 deficiency reduced the bacterial immune response in the} intestine in vivo.
(A) Quantification of weight loss in $\mathrm{YTHDF}^{+/+}$and $\mathrm{YTHDF} 1^{-/-}$littermates after 
ETEC infection; $\mathrm{n}=10$ biological replicates for each genotype; $* * P<0.01$. (B) Survival curve of $\mathrm{YTHDF}^{+/+}$and $\mathrm{YTHDF}^{-/-}$mice following ETEC infection; $\mathrm{n}=10$ biological replicates for both $\mathrm{YTHDF} 1^{+/+}$and $\mathrm{YTHDF}^{-/-}$mice. (C) Representative gross images of colon from YTHDF1 $1^{+/+}$and $\mathrm{YTHDF}^{-/-}$littermates 3 days post-ETEC infection. (D) Cytokines in jejunum and colon of $\mathrm{YTHDF}^{+/+}$and $\mathrm{YTHDF}^{-/-}$mice were determined by ELISA. (E) Histological images of jejunum and colon from YTHDF $1^{+/+}$and $\mathrm{YTHDF}^{-/-}$mice after ETEC infection; $\mathrm{n}=6$ biological replicates for each genotype; scale bar: $50 \mathrm{~mm}$. (F) Immunohistochemistry (IHC) showed infiltration of immune cells in situ in jejunum segments from ETEC-challenged $\mathrm{YTHDF}^{+/+}$and $\mathrm{YTHDF}^{-/-}$mice (brown staining, scale bar $=50$ $\mu \mathrm{m})$. (G) Results from the immunoblot analysis of total YTHDF1, TRAF6, p65 and p-p65 in the jejunum and colon from $\mathrm{YTHDF}^{+/+}$or $\mathrm{YTHDF}^{-/-}$mice. The right panel shows the mRNA level of TRAF6 in the same sample as indicated on the left. Results are presented in relation to those of GAPDH. The data are expressed as the mean \pm s.e.m.; ${ }^{* *} P<0.01 ; \mathrm{n}=6$ biological replicates.

\section{Fig 7. A working model in which YTHDF1 triggers the intestinal immune}

\section{response against bacterial infection.}

DDX60 recruits YTHDF1 to recognize and positively regulate the translation of TRAF6 transcripts to drive intestinal immune responses against bacterial infection. 
bioRxiv preprint doi: https://doi.org/10.1101/2020.05.08.083840; this version posted May 9, 2020. The copyright holder for this preprint

(which was not certified by peer review) is the author/funder, who has granted bioRxiv a license to display the preprint in perpetuity. It is made available under aCC-BY-ND 4.0 International license.

803

804

805 
A

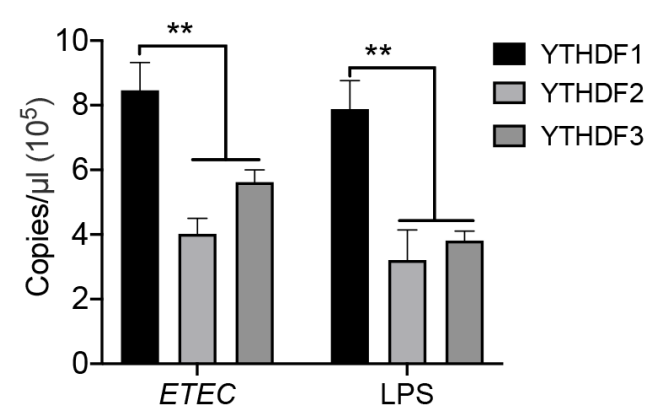

B

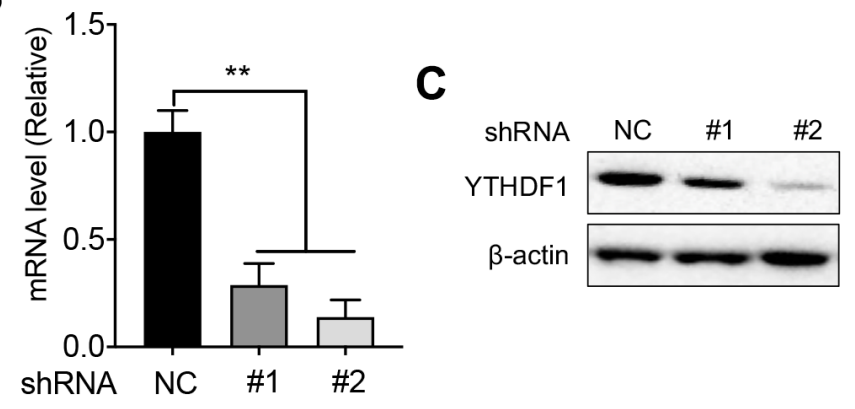

D

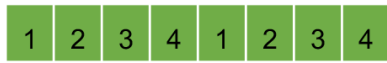

IL-10 IL-12p70

GM-CSF IFN-gamma

TGF-beta TNF-alpha

F

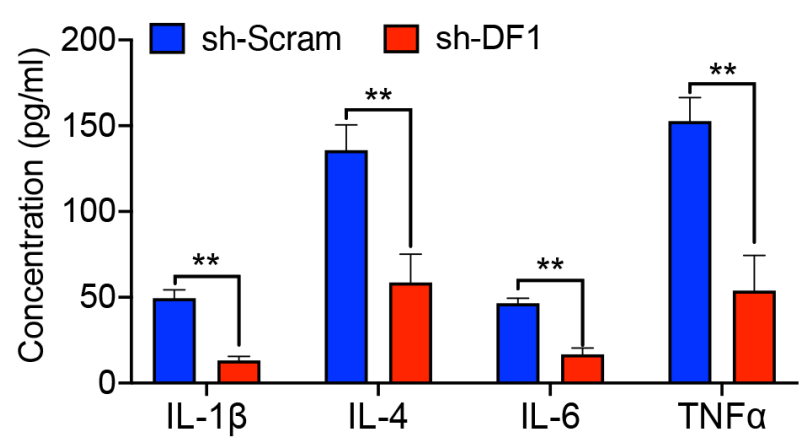

sh-Scram

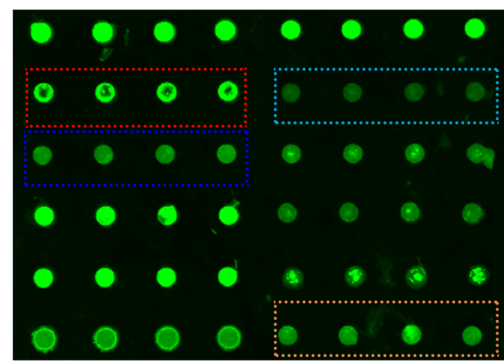

sh-DF1

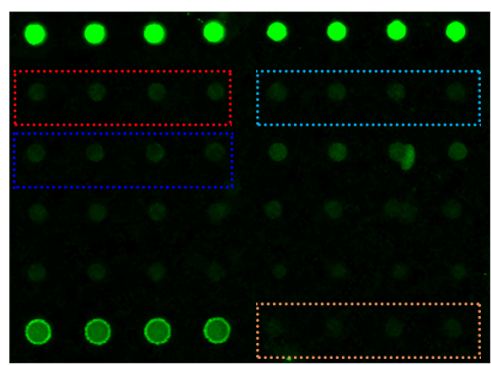

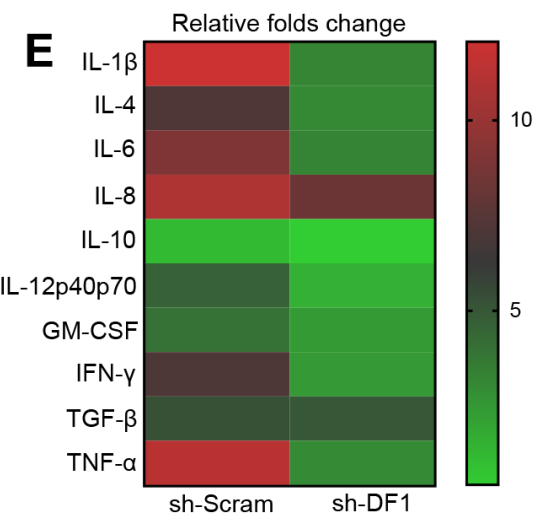

G

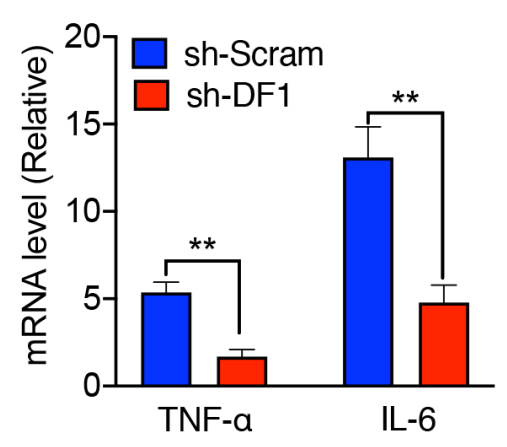

H

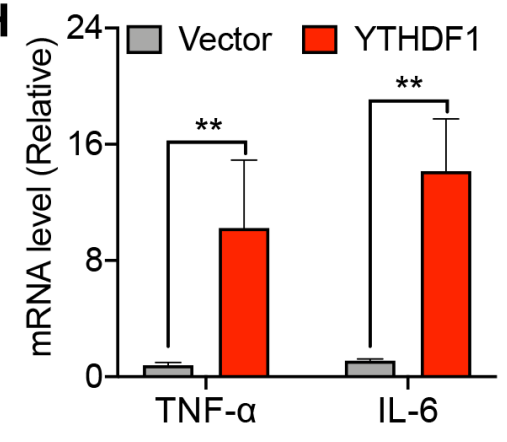




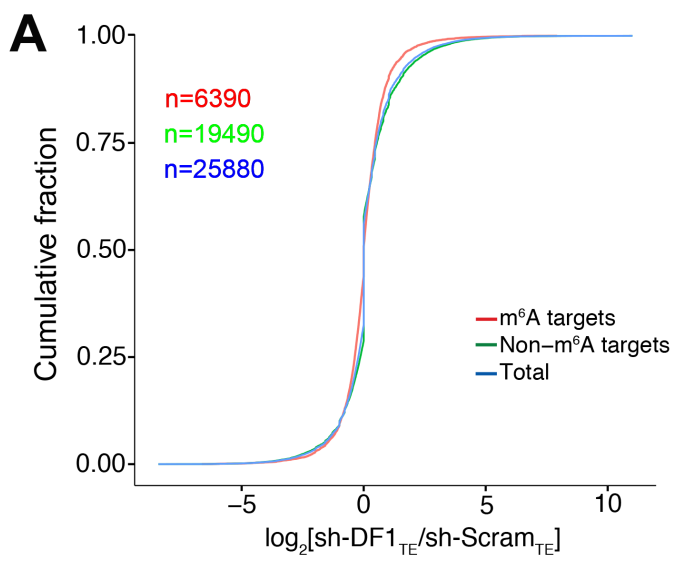

D

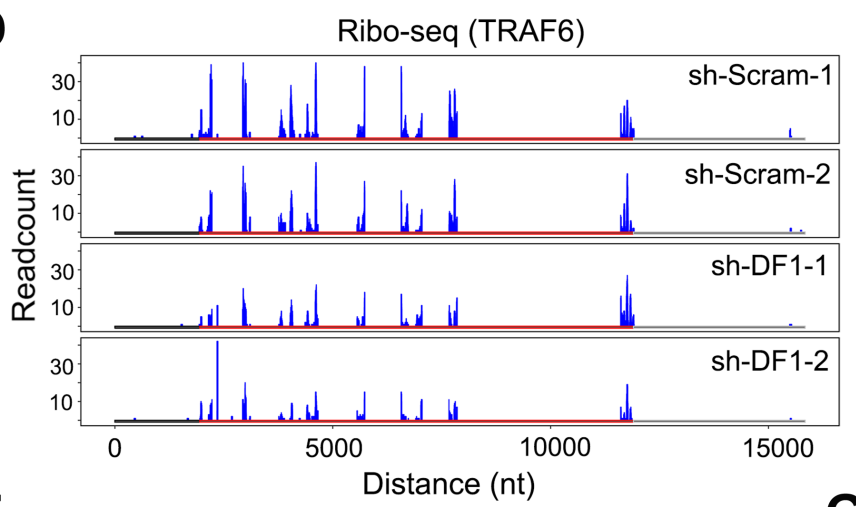

$\mathbf{F}$

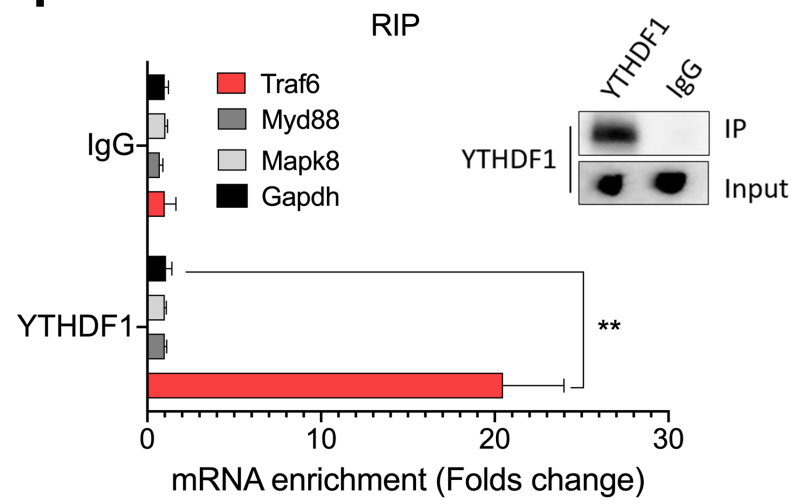

B

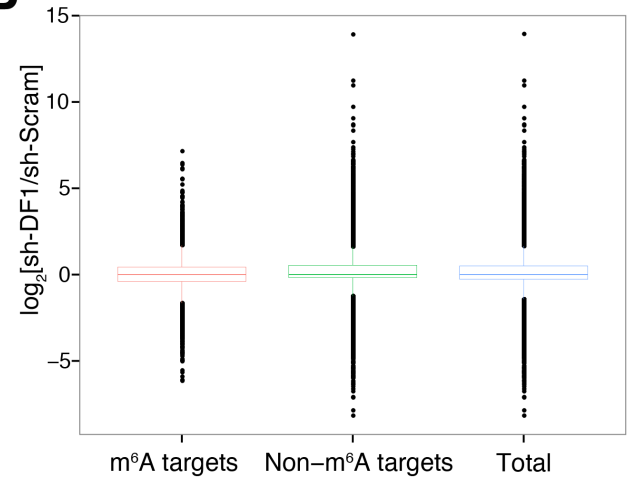

E

TRAF6-Firefly Fluc

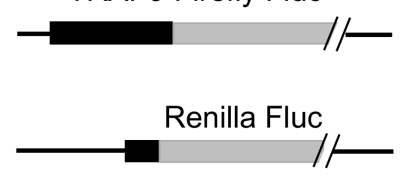

G

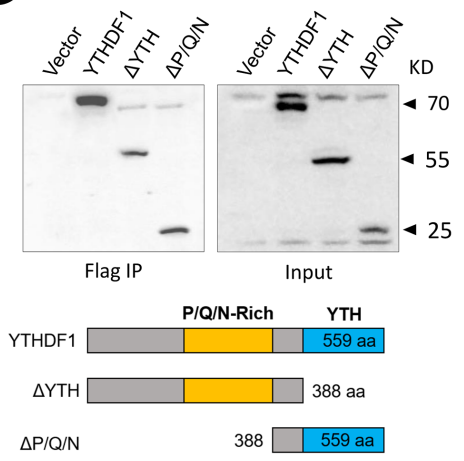

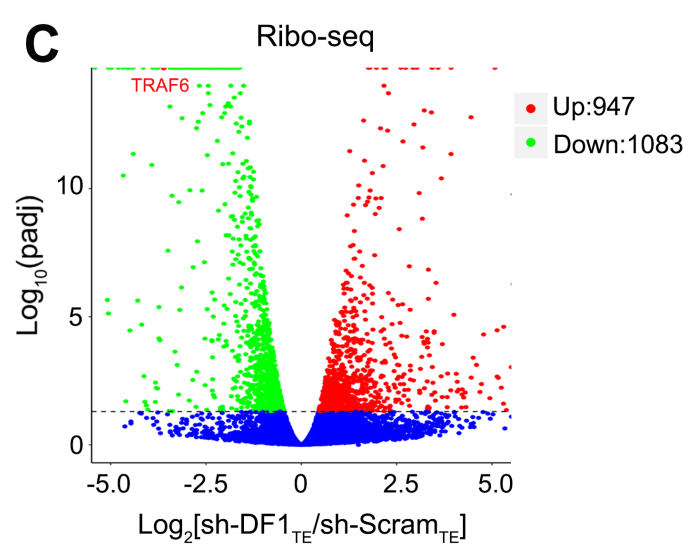

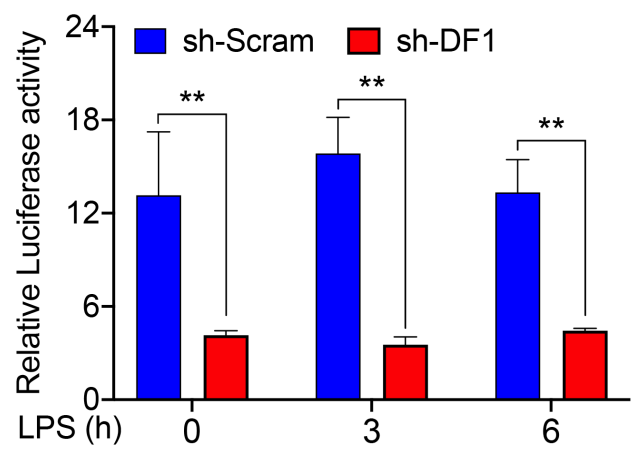

Flag RIP

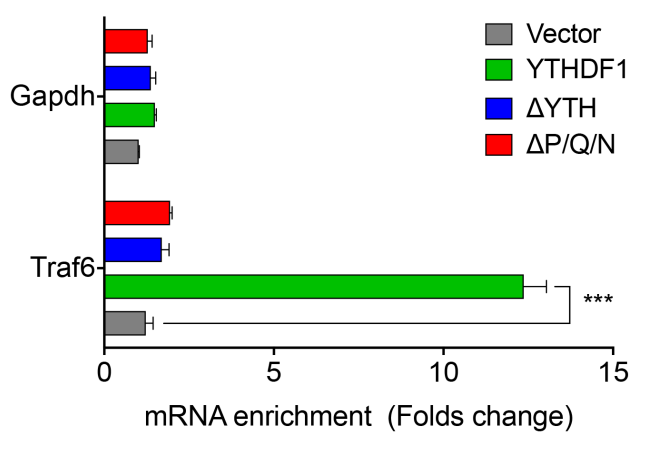


A
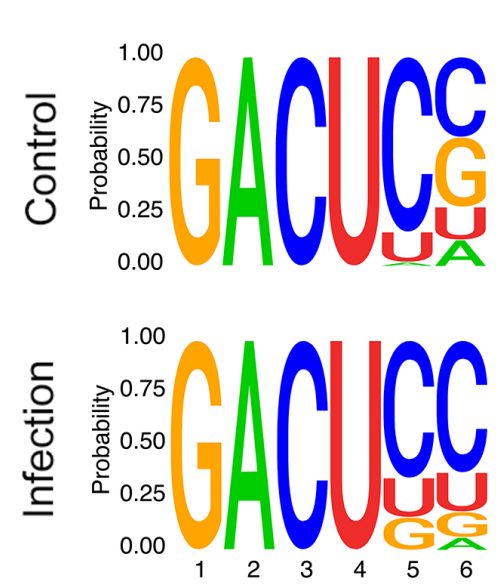

D

Traf6 transgene plasmids

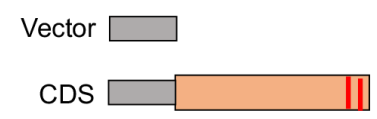

CDS-3'UTR (FL)

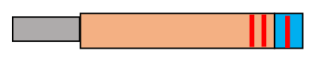

F

Mutant 1 -----GG $\left.\left(m^{6} A\right)\right]$ I----GG $\left.\left(m^{6} A\right)\right]$ C-TAG--Mutant 2 -----GG $\left.\left(m^{6} A\right)\right] C---G G\left(m^{6} A\right)$ I-TAG---

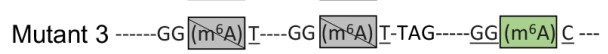
Mutant 4 -----GG $\left.\left(m^{6} A\right) I----G G\left(m^{6} A\right)\right]-T A G-\cdots$ Wild type ----GG $\left(m^{6} A\right) \underline{C}---G G\left(m^{6} A\right) \underline{c}-T A G---G G\left(m^{6} A\right) \underline{c}---$
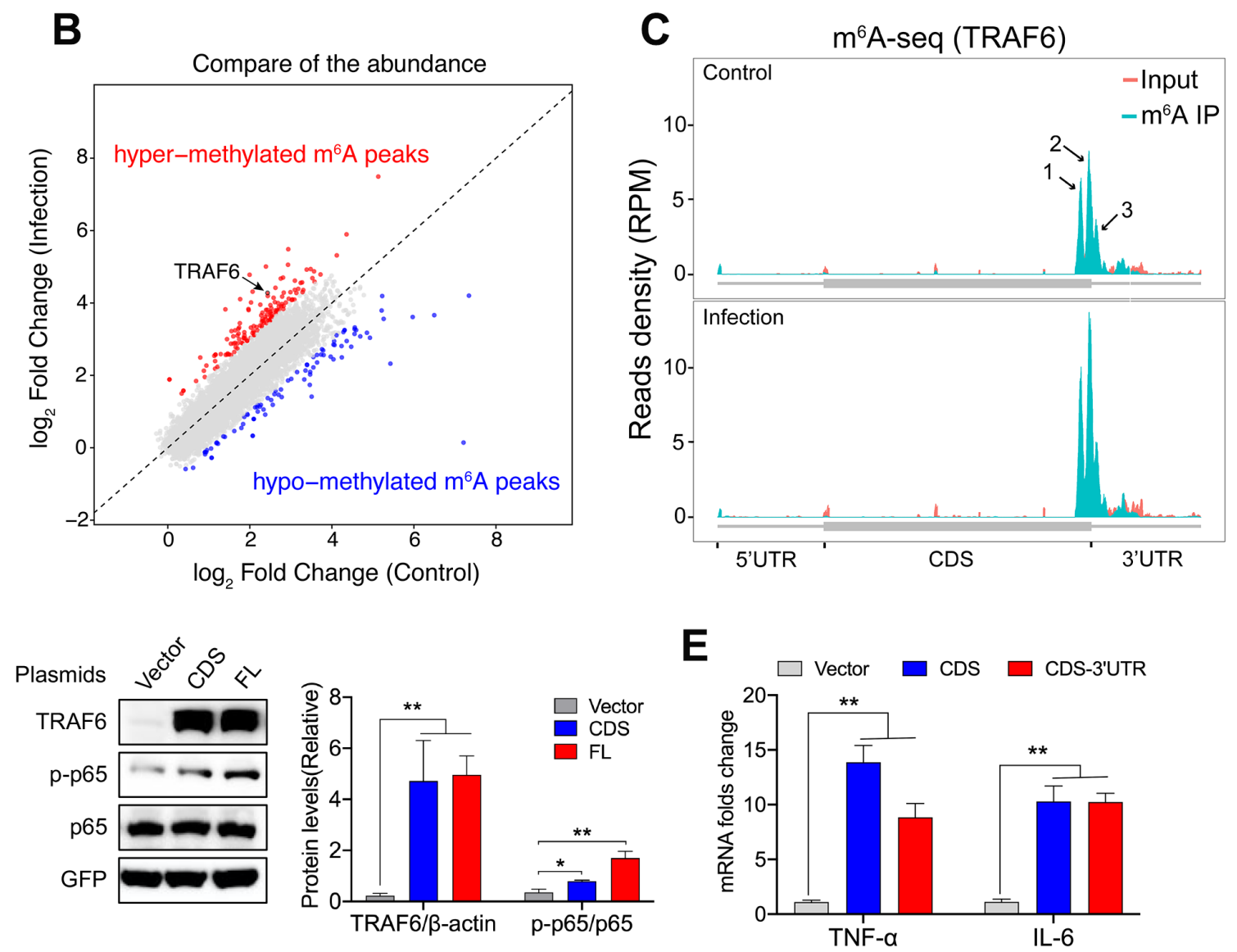

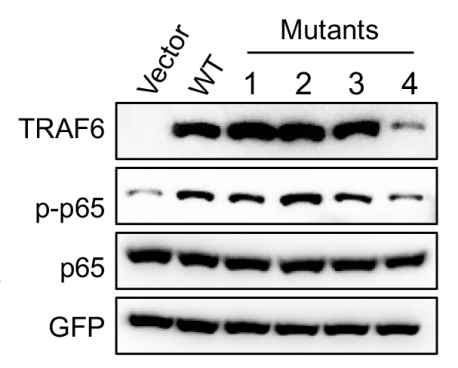

G

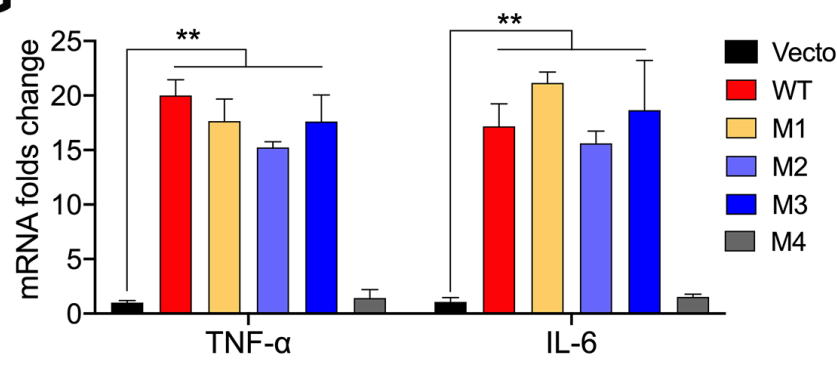




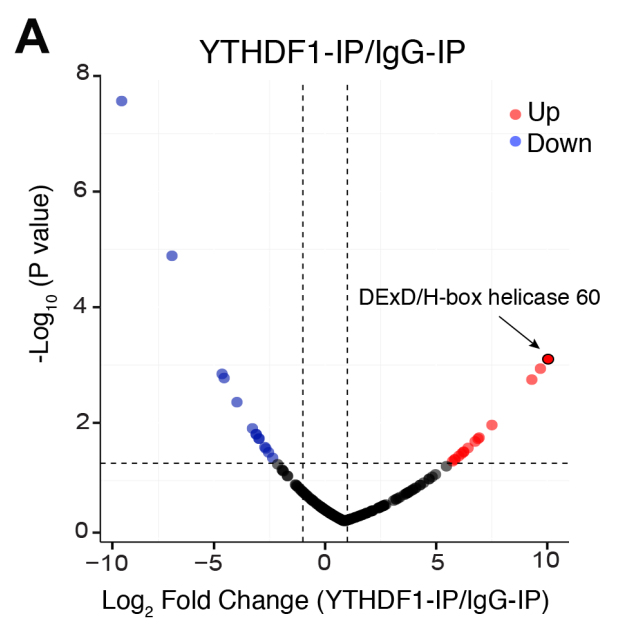

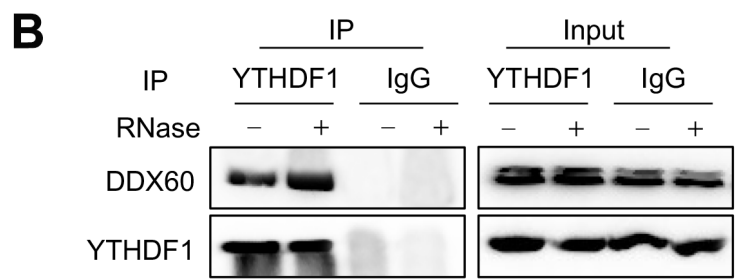

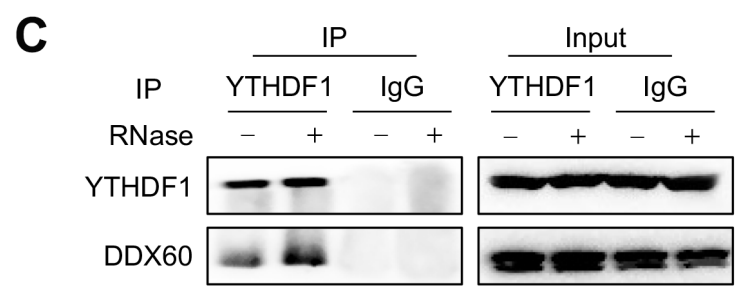

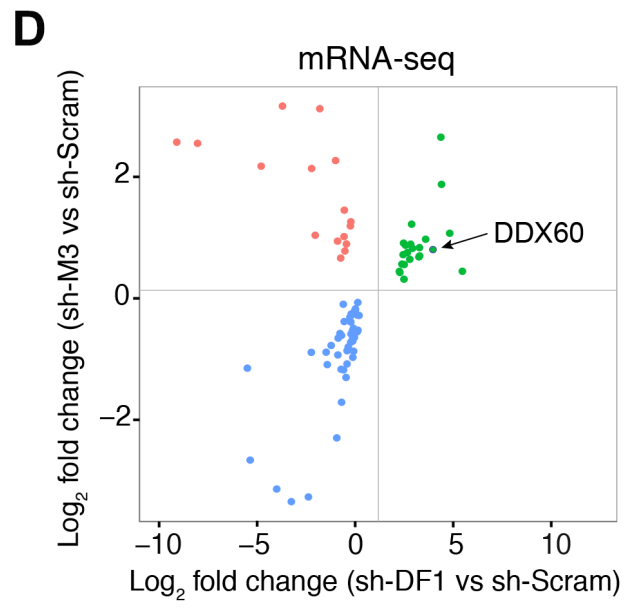

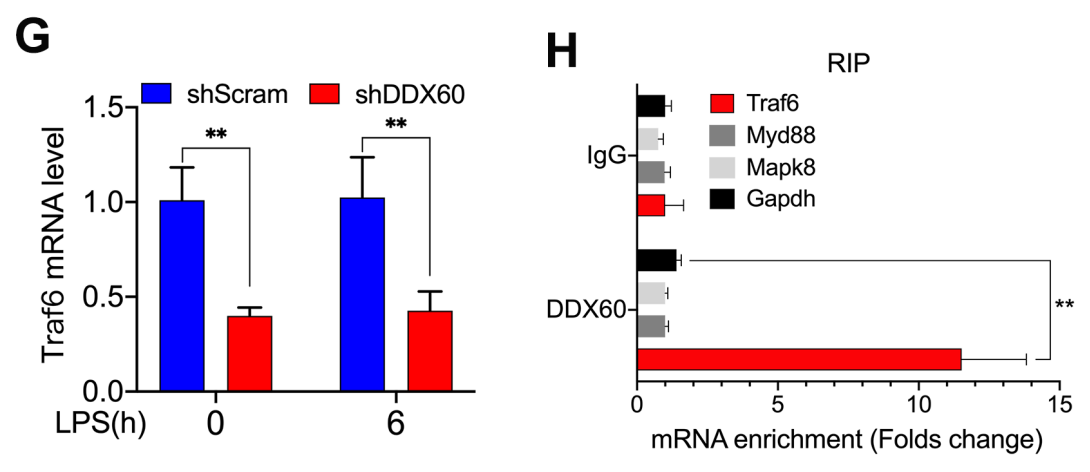

$\mathrm{K}$

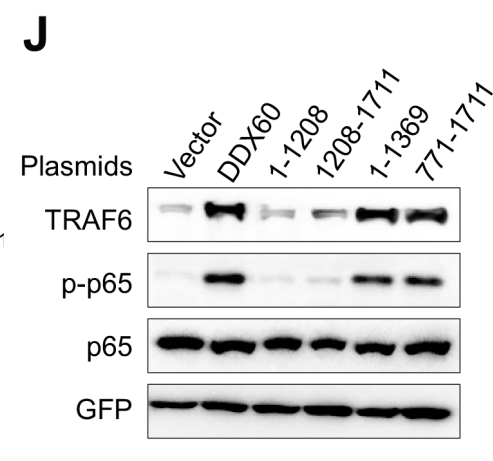

$\square$ Vector $\square$ 1-1208 $\square$ 1-1369

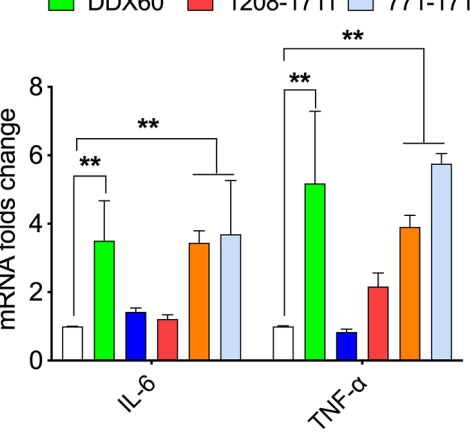


A

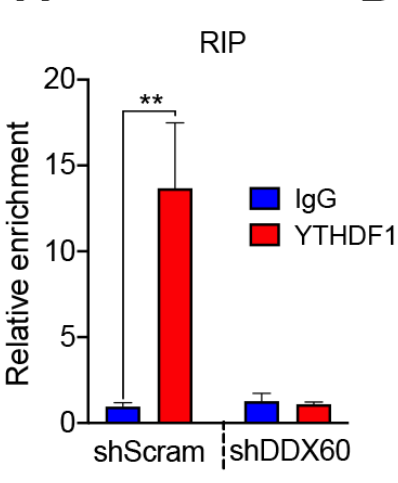

B

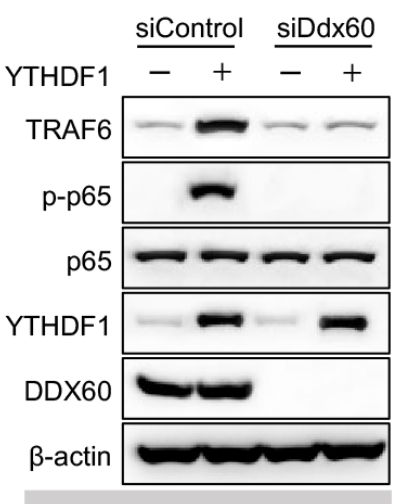

YTHDF1 knockdown cell

C

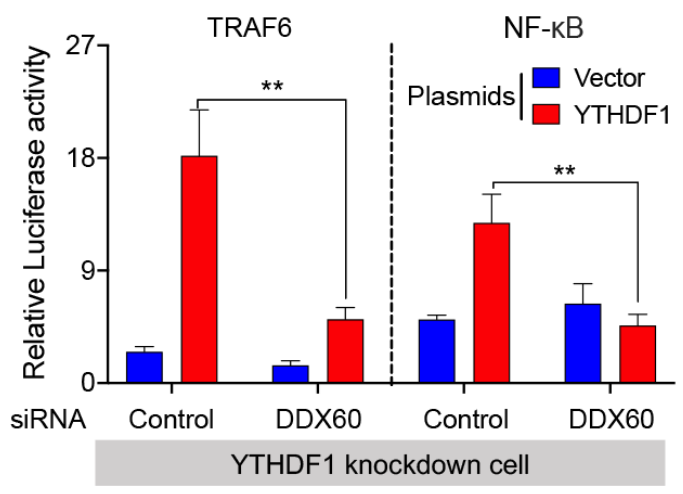

D
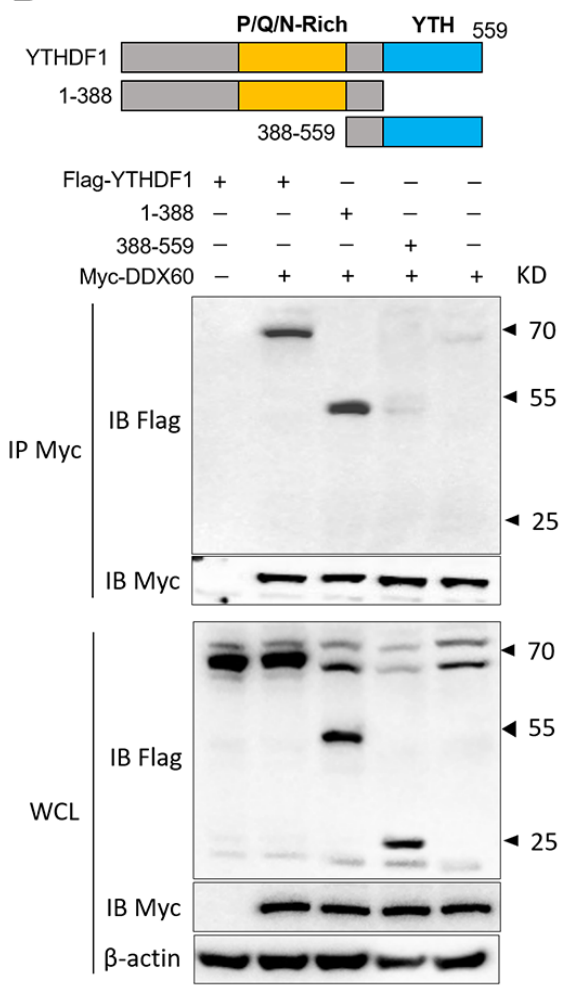

E
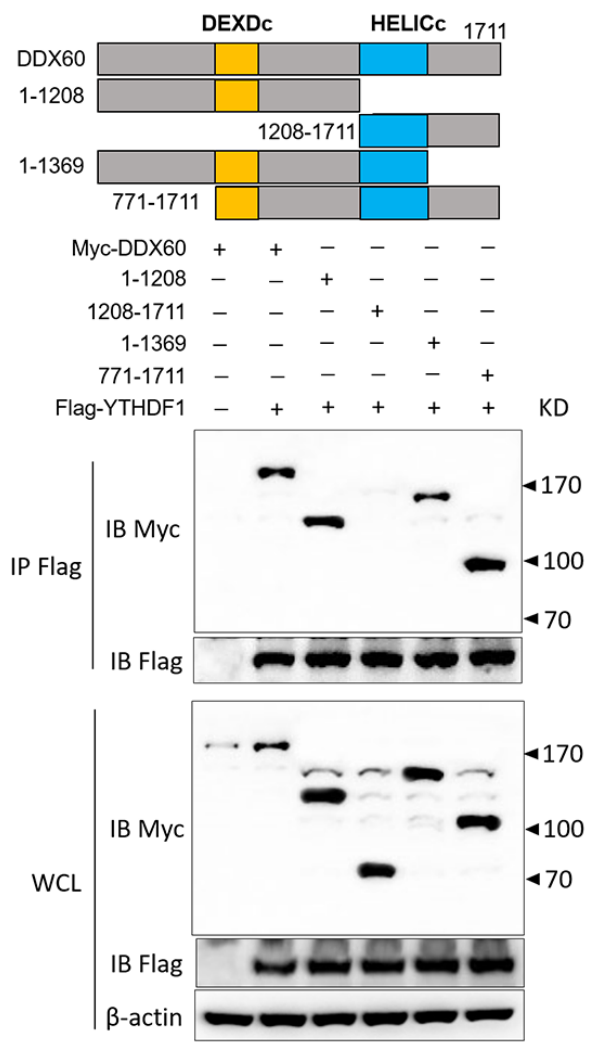

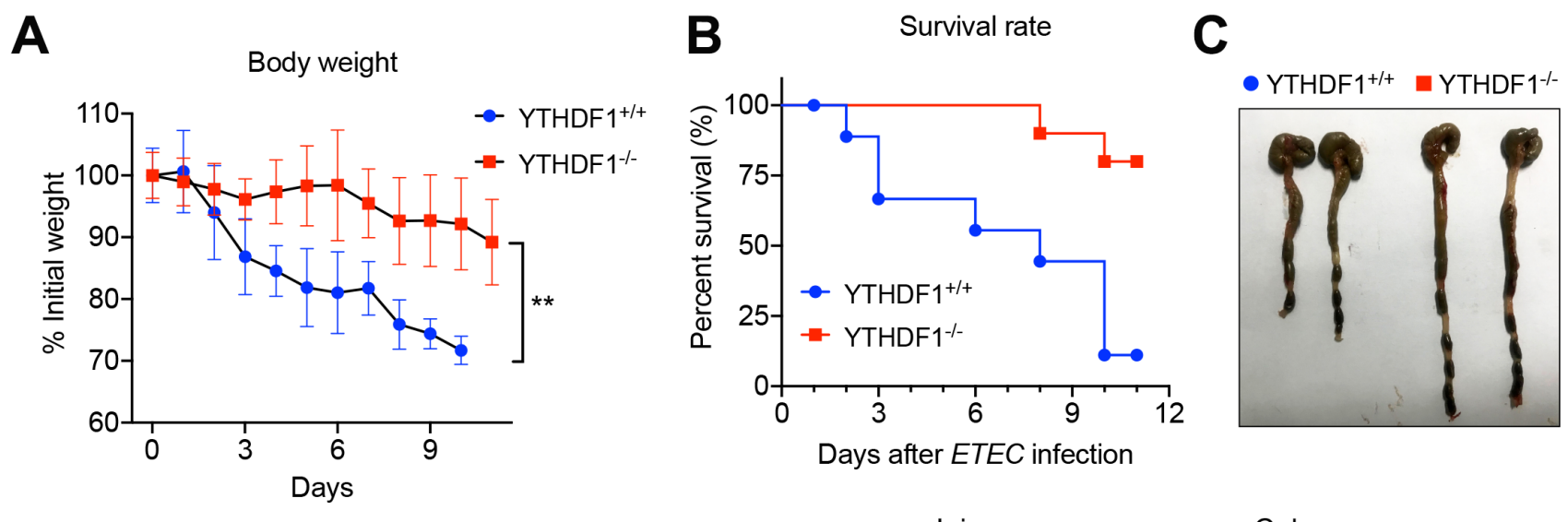

Colon length
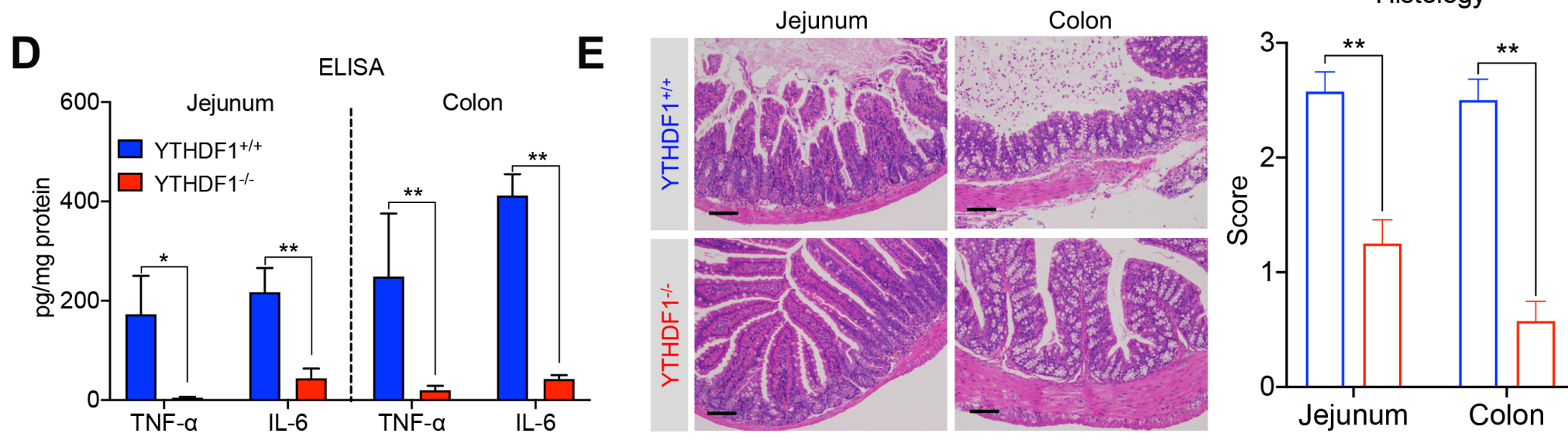

F

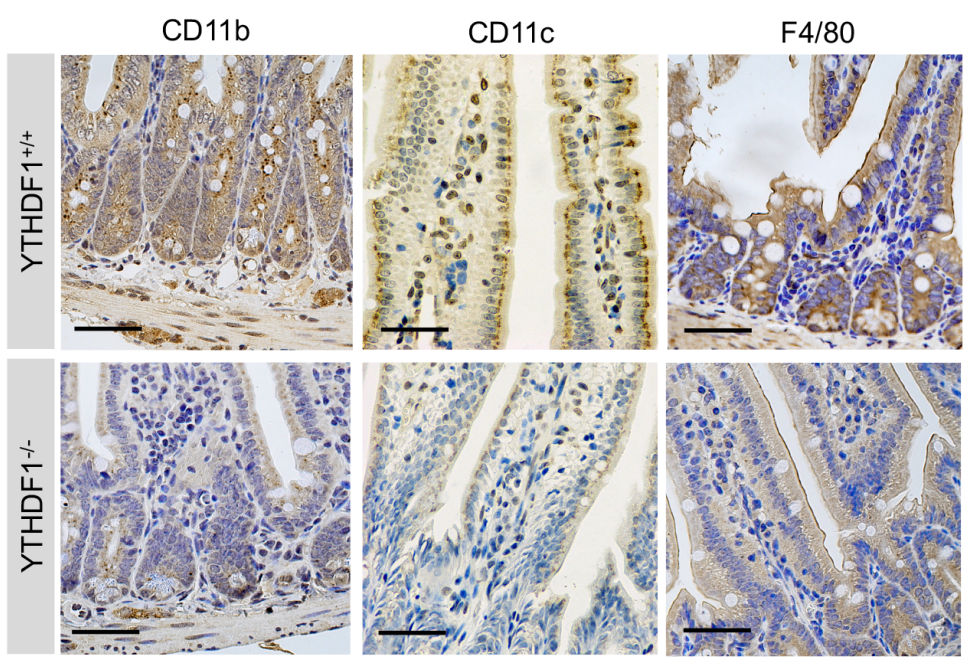

G
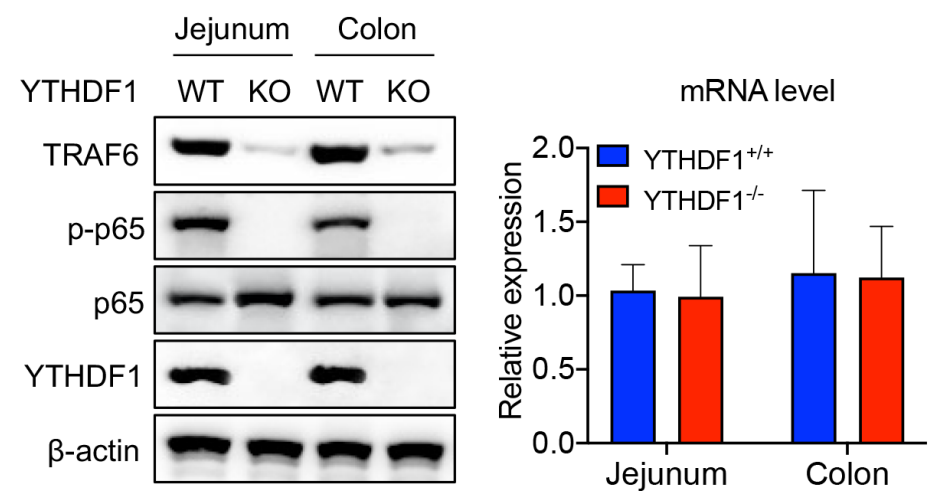
TNF- $\alpha$, IL-6, etc.
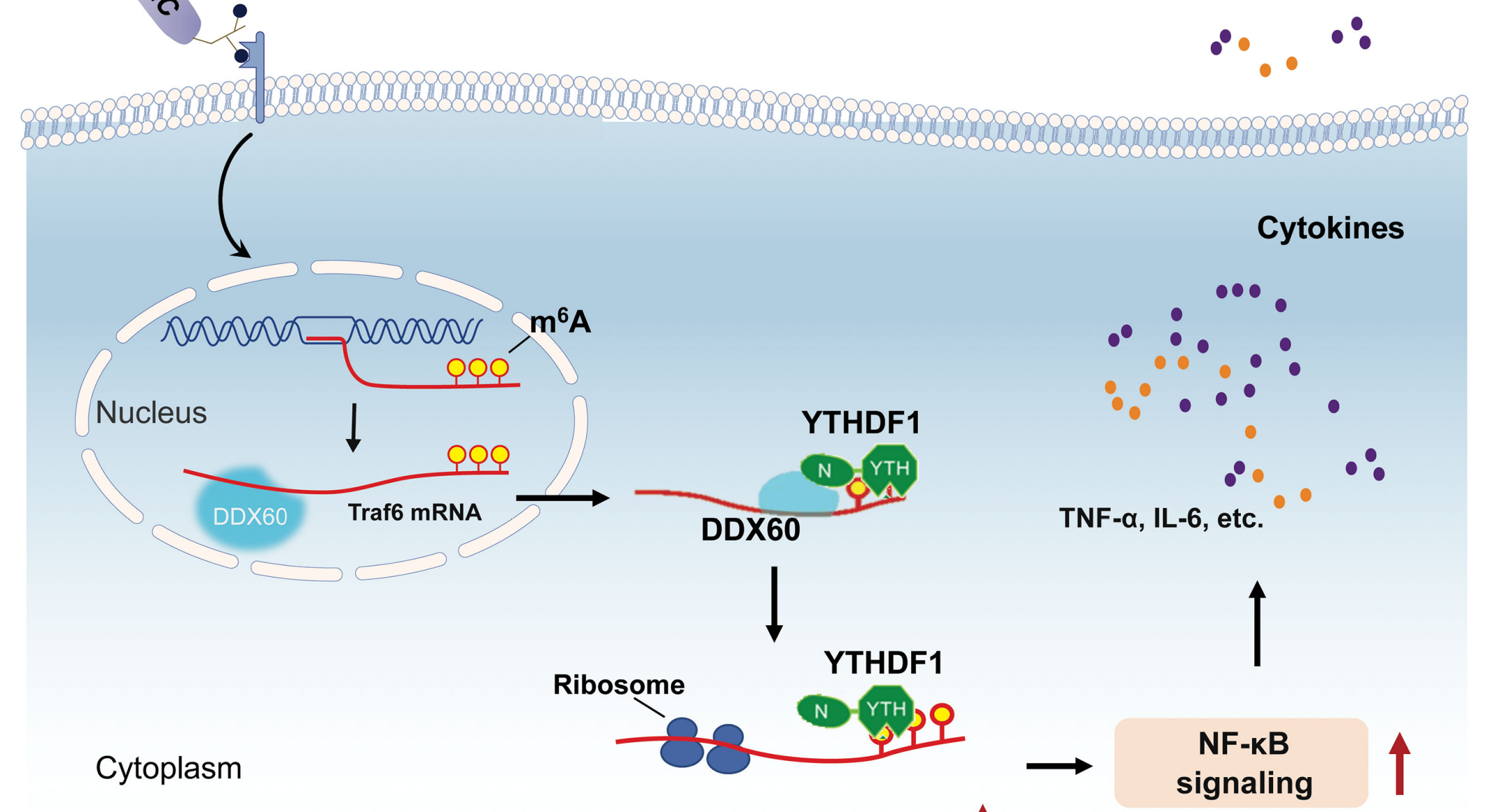

TRAF6 protein synthesis 1

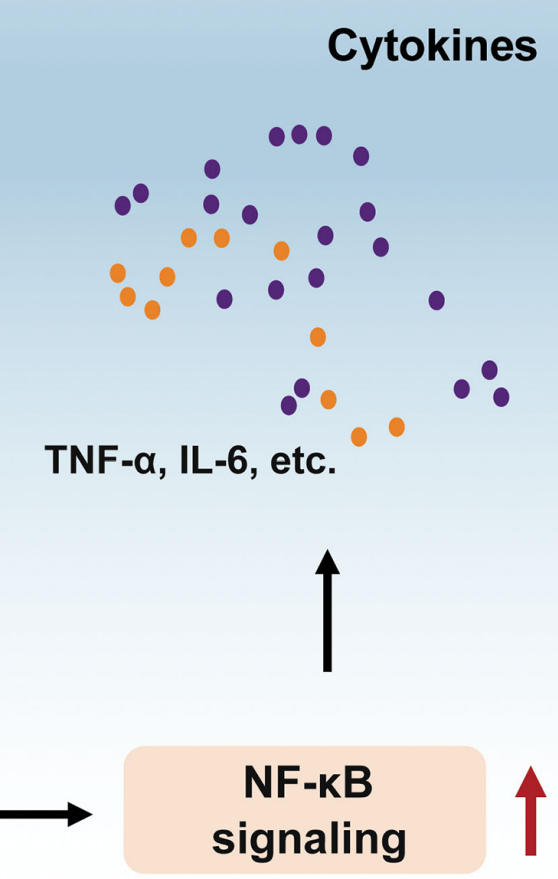

